
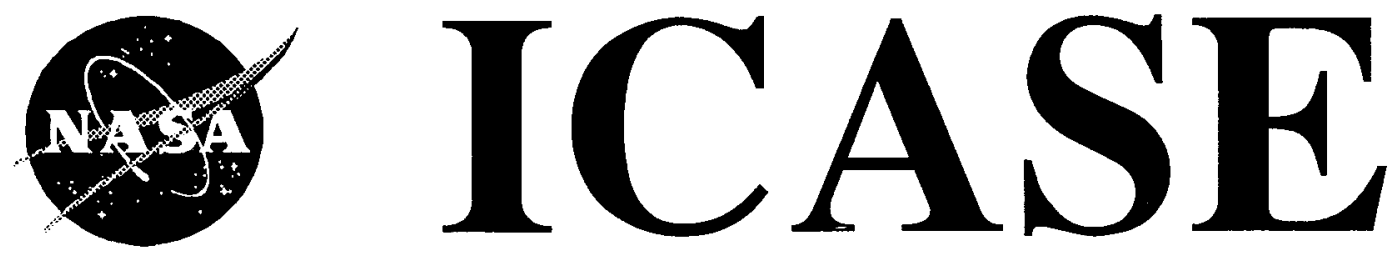

IGNITION DYNAMICS OF A LAMINAR

DIFFUSION FLAME IN THE FIELD OF A VORTEX EMBEDDED IN A SHEAR FLOW

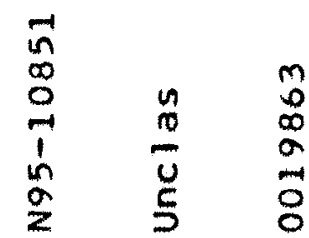

Michele G. Macaraeg

T.L. Jackson

M.Y. Hussaini

Contract NAS1-19480

June 1994

Institute for Computer Applications in Science and Engineering

记岌

委 $z \&$ w

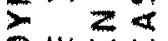

2㟧跑

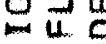

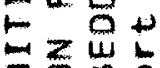

z惒㭊

0 능

$3 \times \propto$

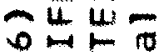

+

\% 0

at

$\rightarrow \sum<3$

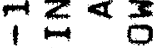

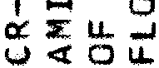

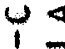

$<0 x$

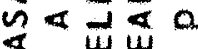

岁出崖出 NASA Langley Research Center Hampton, VA 23681-0001

SRA Operated by Universities Space Research Association 


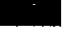




\section{ICASE Fluid Mechanics}

Due to increasing research being conducted at ICASE in the field of fluid mechanics, future ICASE reports in this area of research will be printed with a green cover. Applied and numerical mathematics reports will have the familiar blue cover, while computer science reports will have yellow covers. In all other aspects the reports will remain the same; in particular, they will continue to be submitted to the appropriate journals or conferences for formal publication. 
3

a

$1 \mathrm{a}$

0

a

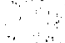

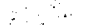

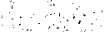

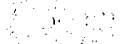

.

$\therefore \quad$

$\therefore$ 


\title{
IGNITION DYNAMICS OF A LAMINAR DIFFUSION FLAME IN THE FIELD OF A VORTEX EMBEDDED IN A SHEAR FLOW
}

\author{
Michéle G. Macaraeg \\ NASA Langley Research Center \\ Hampton, Virginia 23681-0001 \\ T.L. Jackson and M.Y. Hussaini \\ Institute for Computer Applications in Science and Engineering \\ NASA Langley Research Center \\ Hampton, Virginia 23681-0001
}

\begin{abstract}
The role of streamwise-spanwise vorticity interactions that occur in turbulent shear flows on flame/vortex interactions is examined by means of asymptotic analysis and numerical simulation in the limit of small Mach number. An idealized model is employed to describe the interaction process. The model consists of a one-step, irreversible Arrhenius reaction between initially unmixed species occupying adjacent half-planes which are then allowed to mix and react in the presence of a streamwise vortex embedded in a shear flow. It is found that the interaction of the streamwise vortex with shear gives rise to small-scale velocity oscillations which increase in magnitude with shear strength. These oscillations give rise to regions of strong temperature gradients via viscous heating, which can lead to multiple ignition points and substantially decrease ignition times. The evolution in time of the temperature and mass-fraction fields is followed, and emphasis is placed on the ignition time and structure as a function of vortex and shear strength.
\end{abstract}

The second and third authors were supported by the National Aeronautics and Space Administration under NASA Contract Number NAS1-19480 while in residence at the Institute for Computer Applications in Science and Engineering, NASA Langley Research Center, Hampton, VA 23681. 
8 a 


\section{INTRODUCTION}

The presence of streamwise vortices is now very well established in wall-bounded shear flows as well as free shear flows either in transitional state or fully developed turbulent state. The investigations of Klebanoff, Tidstrom and Sargent (1962), Wray and Hussaini (1984), and Zang and Hussaini (1987) provide the evidence for the existence of streamwise vortices in low-speed boundary layers undergoing laminarturbulent transition. Such vortices have been observed in the buffer region of the turbulent boundary layers by Blackwelder and Eckelmann (1979) for instance. The review of Willmarth (1975) cite some earlier observations of these phenomena. In free shear flows, the experimental studies (e.g., Lasheras and Choi (1988) and the references therein), theoretical studies (e.g., Lin and Corcos (1984) and the references cited therein), and the direct numerical simulations (e.g., Metcalf, et al. (1987) and Ashurst and Meiburg (1988)) have provided some insight into the origin and evolution of these streamwise vortices.

The evolution of a shear flow in the presence of streamwise vortices, or conversely, the dynamics of vortices stretched by shear flow is of fundamental interest in fluid mechanics. A physically and geometrically simple model of such interactions between spanwise and streamwise vorticity appears to have been first proposed by Pearson and Abernathy (1984). Their model assumes an incompressible mean flow with uniform shear which is then disturbed by the introduction of an Oseen vortex with its axis in the streamwise direction. Corcos (1988) has studied this problem by a systematic asymptotic approach with a focus and range different from that of Pearson and Abernathy. He showed that the streamwise velocity component underwent severe distortions in a region of thickness $v^{1 / 3}$ as a result of the streamwise vortex. These distortions were damped out in the viscous core region, which is of size $v^{1 / 2}$. This model, however, is restricted to the analysis of streamwise structures of turbulent non-reacting shear flows. We shall now focus our attention to reacting flows.

An important problem in the general area of non-premixed combustion is the study of chemical reactions in turbulent reacting flows. However, such flows are difficult to analyze and few theoretical studies have been conducted. One such theoretical investigation, which forms the genesis of our model and is to be described in more detail later, is that of Marble (1985), who developed a simple model problem which locally describes an established thin flame that is being wrapped up by a small scale eddy. However, there are certain important aspects, such as ignition and flame structure, which were not included within the framework of the Marble problem.

We have completed a systematic analysis of the ignition process and the subsequent flame structure of diffusion flames in vortex dominated flows using asymptotics and direct numerical simulation in the absence of a shear flow (Macaraeg, Jackson and Hussaini, 1992). We considered a constant-density, one-step, irreversible Arrhenius reaction between initially unmixed species of fuel and oxidizer occupying adjacent half-planes, which were then allowed to mix and react in the presence of a single point vortex. Using large activation energy asymptotics, particular attention was paid to the ignition regime as a function of the vortex Reynolds number. For near equal initial temperatures of the fuel and oxidizer and expanding the temperature about its inert value, it was shown that the overall ignition time decreases slightly and then asymptotes to a finite value as the vortex Reynolds number is increased from zero. For finite values of the activation energy, a direct numerical solution was used to study post-ignition events. First, a hot spot develops within the viscous core of the vortex (ignition) and progresses rapidly into a circular flame which grows in time according to a similarity rule. Next, a tribrachial flame configuration evolves at infinite distance to the right 
and left of the origin. The diffusion flames emanating from this configuration then move toward and finally merge with the flame in the reacted core region. We have recently extended the ignition study to flows with counter rotating vortices (Kozusko, Macaraeg and Jackson, 1993).

The above mentioned problem of a flame interacting with a single point vortex is supposed to model certain fundamental mechanisms of turbulent diffusion flames which form the basis of many propulsion devices. Since acoustic waves are always present in these combustion systems, the role of acoustics in such flame/vortex interactions was investigated (Jackson, Macaraeg and Hussaini, 1993). The acoustic field was considered to be a spatially uniform but time dependent pressure wave of small amplitude. This pressure wave was then taken to have the sinusoidal form $P(t)=P_{A} \sin (\omega t+\Phi)$, where $P_{A}$ is the amplitude, $\omega$ is the frequency and $\Phi$ is the phase, and was regarded as a single Fourier mode of a more general pressure wave. It was concluded that for low frequencies, ignition is accelerated or significantly delayed depending on the phase, while for moderate to high frequencies, ignition is always enhanced independent of phase. Finally, an approximation for the ignition time was found by considering an equivalent homogeneous problem in which spatial variations are ignored. This leads to the following implicit formula for the "homogeneous" ignition time $t_{i g}^{H}$, given by

$$
\int_{0}^{t_{i g}^{H}} e^{P(\tau)} d \tau=t_{i g}^{0},
$$

where $t_{i g}^{0}$ is the ignition time in the absence of a pressure disturbance. Thus, this implicit formula relates the ignition time when pressure disturbances are absent to the homogeneous ignition time when pressure disturbances are present. It was found that this homogeneous ignition time predicts the ignition trends very well, and therefore was proposed as a good engineering approximation for obtaining the ignition times for the more complicated flow fields.

The above mentioned ignition studies have been confined to vortices evolving in an otherwise constant flow. However, shear is an important attribute of turbulent flows. Thus, the purpose of the present study is to extend the above analysis so as to investigate the effects of the interaction between a streamwise vortex and spanwise vorticity induced by the shear on ignition. We assume a simplified kinetic model consisting of a one-step, irreversible Arrhenius reaction between unmixed species occupying adjacent halfplanes which are then allowed to mix and react in the presence of a streamwise vortex embedded in a shear flow. We remark here that any realistic modeling of complex kinetics will necessitate a full numerical solution. Then questions arise as to the reaction rates and their dependence on temperature and the relative importance of various reactions which entail thus rather large uncertainties. In a mathematical treatment of combustion, the kinetic model has to be necessarily simple. The one-step irreversible Arrhenius model has been extensively used with significant success in the study of low-speed combustion (e.g., Buckmaster and Ludford, 1982). This model appears to cover the essential physics of the problem. In the last decade or so, the asymptotic studies of combustion based on this model have significantly enhanced our understanding of ignition, of flame stability and of diffusion flame structure in low subsonic flows. There is as yet no reason to believe that this model will not play an equally significant role in enhancing our understanding of supersonic combustion. In any event, this idealization makes the problem amenable to asymptotic analysis and thus provides a semi-analytical solution. The results can then certainly verify and in turn be verified by full numerical simulations. An idealized model is now employed to describe the interaction process: the 
streamwise vortex, assumed to be infinite in extent, interacts in time with the streamwise velocity component associated with the spanwise vorticity. Initially, the streamwise velocity is assumed to be a linear shear profile and the streamwise vortex resides along the fuel-oxidant interface. The reactants are then allowed to mix and react. Emphasis is placed on ignition time and structure. In Section 2 the basic equations and assumptions are presented. Section 3 contains the ignition analysis and results, and the conclusion is given in Section 4.

\section{PROBLEM FORMULATION}

In this section the problem for the time evolution of initially unmixed species occupying adjacent half-planes which are then allowed to mix and react in the presence of a vortex embedded in a shear flow (Figure 1) is formulated. We assume that the flow is independent of the downstream direction $x$ for all time. Although it is not strictly true for any real flow, it is consistent with experimental observations that streamwise vortical structures in turbulent flows are essentially aligned with the flow and are of great streamwise extent. Since we are interested in flows with vortical motion, the basic equations are transformed into cylindrical coordinates $(y, z) \rightarrow(r, \theta)$ by the transformations $y=r \cos \theta$ and $z=r \sin \theta$. If we define $g$ to be the radial velocity and $h$ to be the axial velocity, i.e., $v=g \cos \theta-h \sin \theta$ and $w=g \sin \theta+h \cos \theta$, then the governing non-dimensional equations in cylindrical coordinates, neglecting all dependency in $x$, reduce to

$$
\begin{gathered}
\rho T=P, \\
\rho_{t}+(\rho g)_{r}+\frac{1}{r}(\rho h)_{\theta}+\frac{\rho g}{r}=0, \\
\rho\left[u_{t}+g u_{r}+\frac{h}{r} u_{\theta}\right]=\frac{1}{\operatorname{Re}} \nabla^{2} u, \\
\rho\left[g_{t}+g g_{r}+\frac{h}{r} g_{\theta}-\frac{h^{2}}{r}\right]+\frac{P_{r}}{\gamma M^{2}}=\frac{1}{\operatorname{Re}}\left[\nabla^{2} g-\frac{g}{r^{2}}-\frac{2}{r^{2}} h_{\theta}+\frac{1}{3} K_{r}\right], \\
\rho\left[h_{t}+g h_{r}+\frac{h}{r} h_{\theta}+\frac{g h}{r}\right]+\frac{1}{\gamma M^{2}} \frac{P_{\theta}}{r}=\frac{1}{\operatorname{Re}}\left[\nabla^{2} h-\frac{h}{r^{2}}+\frac{2}{r^{2}} g_{\theta}+\frac{1}{3 r} K_{\theta}\right], \\
\rho\left[T_{t}+g T_{r}+\frac{h}{r} T_{\theta}\right]-\frac{\gamma-1}{\gamma}\left[P_{t}+g P_{r}+\frac{h}{r} P_{\theta}\right]=\frac{1}{\operatorname{Re} P r} \nabla^{2} T+\frac{(\gamma-1) M^{2}}{\operatorname{Re}} \Phi+\beta \Omega, \\
\rho\left[F_{j, t}+g F_{j, r}+\frac{h}{r} F_{j, \theta}\right]=\frac{1}{\operatorname{Re} S c} \nabla^{2} F_{j}-\Omega, \\
\Omega=\tau D a \rho F_{1} F_{2} e^{-Z e / T}, \\
j=1,2,
\end{gathered}
$$


where $\nabla^{2}$ is the two-dimensional Laplacian operator in cylindrical coordinates, $\Phi$ is the viscous dissipation term, and $K=g_{r}+g / r+h_{\theta} / r$. Here, $(u, g, h)$ are the velocity components in the $(x, r, \theta)$ directions, respectively; $\rho$ is the density; $T$ is the temperature; $P$ is the pressure; and $F_{1}$ and $F_{2}$ the mass fractions of the fuel and oxidizer, respectively. The chemical model is assumed to be a one-step, irreversible Arrhenius reaction. The nondimensional parameters appearing above are the Schmidt number $S c=v / D$ assumed equal for both species with $D$ the species diffusion coefficient and $v$ the kinematic viscosity; the Prandtl number $\operatorname{Pr}$; the Reynolds number $\operatorname{Re}=L_{v} V_{v} / v$ where $L_{v}$ and $V_{v}$ are characteristic length and velocity scales of the vortex; the Zeldovich number $Z e=E /\left(R^{o} T_{\infty}\right)$ with $E$ the dimensional activation energy and $R^{o}$ the universal gas constant; the Damkohler number $D a=t_{d} / t_{c}$ defined as the ratio of the characteristic diffusion time scale $t_{d}$ to the characteristic chemical time scale $t_{c} ; \beta$ the heat release per unit mass of $F_{1} ; \gamma$ the ratio of specific heats; the Mach number $M=V_{v} / a_{\infty}$ where $a_{\infty}$ is the characteristic speed of sound; and finally $\tau=t_{v} / t_{d}$ is the ratio of the time scale of the vortex $t_{v}$ to that of diffusion. The density, temperature, pressure and mass fractions are nondimensionalized by their initial values $\rho_{\infty}, T_{\infty}, P_{\infty}=\rho_{\infty} R^{o} T_{\infty}$ and $F_{1, \infty}$, respectively. Lengths are referred to $L_{v}$, a characteristic lengthscale of the vortex, such as the core size, and velocities are referred to $V_{v}$, the reference tangential velocity. The actual choice for these scales is given in the next section. The relevant time scale is therefore $t_{v}=L_{v} / V_{v}$. The system (2.1) must be solved subject to appropriate initial and boundary conditions chosen to represent a streamwise Oseen vortex evolving in a shear flow.

\section{IGNITION}

At time $t=0$, the reaction rate is exactly zero owing to the product $F_{1} F_{2}=0$. For $t>0$, the fuel and oxidizer begin to mix by diffusion, as well as by convection due to the presence of the vortex, and the reaction rate is no longer zero. For small time, it can thus reasonably be assumed that the effect of the reaction on the overall flow field is small. The solution for small time in which the reaction rate term is neglected is known as the inert or chemically frozen solution, and will be denoted by the superscript I. In what follows, we only consider the case of near equal initial temperatures and small Mach numbers, and take the asymptotic limit of large Zeldovich number (for the simplified hydrogen-oxygen system $2 \mathrm{H}_{2}+\mathrm{O}_{2} \rightarrow 2 \mathrm{H}_{2} \mathrm{O}$ in the presence of an inert $N_{2}$, the dimensional activation energy $E$ lies in the range $\mathrm{O}(7-40 \mathrm{kcal} / \mathrm{mole})$ yielding $Z e=5-20$ for $T_{\infty}$ in the range $300-2000^{\circ} \mathrm{K}$; e.g., Glassman, 1977; also, Drummond and Mukunda, 1988 , in their numerical work on reacting mixing layers uses $7200 \mathrm{cal} / \mathrm{mole})$. For large activation energies (and hence large Zeldovich numbers), this assumption leads to the inert solutions $T^{I}=\rho^{I}=1+O\left(Z e^{-1}\right)$, provided that the Mach number is no larger than $Z e^{-1 / 2}$ so that the viscous dissipation term can be neglected to leading order. For larger Mach numbers the temperature field can no longer be considered uniform to leading order since heating must take place by means of the dissipation term. The $O\left(Z e^{-1}\right)$ term is included to allow for small initial temperature differences across the interface. Thus, the leading-order inert solution corresponds to the constant density approximation prevalent in the combustion literature. As time increases, more of the combustible mixes until, at some finite time, a thermal explosion occurs characterized by significant departure from the inert. To analyze the ignition process, we determine the effect of the growing reaction rate by expanding about the inert solution as

$$
T=1+Z e^{-1} T_{1}+O\left(Z e^{-2}\right), \quad \rho=1+Z e^{-1} \rho_{1}+O\left(Z e^{-2}\right), \quad F_{j}=F_{j}^{I}+O\left(Z e^{-1}\right)
$$




$$
(u, g, h)=\left(u^{I}, g^{I}, h^{I}\right)+O\left(Z e^{-1}\right), \quad P=1+\gamma M^{2}\left[P^{I}+O\left(Z e^{-1}\right)\right]
$$

and take the asymptotic limit $Z e \rightarrow \infty$. The viscous dissipation term in (2.1f) in this limit is of the order $M^{2} Z e$, and for a proper balance of the energy equation we set

$$
M=M_{o} / \sqrt{Z e}
$$

Thus, solutions are also sought in limit of small Mach numbers. A comment about the size of the Mach number and the relative importance of the viscous dissipation term in the energy equation is in order here. For typical combustion systems the Mach numbers are of the order $\mathrm{O}\left(10^{-3}\right)$, and so the role of viscous heating is negligible. Here, however, we have in mind propulsion devices in high speed flows. As such, the Mach number within the mixing region can be relatively large. For example, in their study of compressible viscous vortices, Colonius et al. (1991) report Mach numbers in the range of 0.0625 to 0.67 (the definition of their Mach number is the same as used here). In our work, we have taken $M_{0}=\sqrt{Z e} M$ with $M_{0}$ lying in the range (0.3-0.7), corresponding to Mach numbers of 0.01 to 0.22 , respectively, for a typical value of $Z e=10$. These values are well within physical ranges. Thus, in these flow situations, viscous heating can indeed play a major role in the ignition dynamics.

The leading-order equations are given by

$$
\begin{gathered}
\rho_{1}+T_{1}=\gamma M_{o}^{2} P^{I}, \\
g_{r}^{I}+\frac{1}{r} h_{\theta}^{I}+\frac{g^{I}}{r}=0 \\
u_{t}^{I}+g^{I} u_{r}^{I}+\frac{h^{I}}{r} u_{\theta}^{I}=\frac{1}{\operatorname{Re}} \nabla^{2} u^{I}, \\
g_{t}^{I}+g^{I} g_{r}^{I}+\frac{h^{I}}{r} g_{\theta}^{I}-\frac{h^{I^{2}}}{r}+P_{r}^{I}=\frac{1}{\operatorname{Re}}\left(\nabla^{2} g^{I}-\frac{g^{I}}{r^{2}}-\frac{2}{r^{2}} h_{\theta}^{I}\right), \\
h_{t}^{I}+g^{I} h_{r}^{I}+\frac{h^{I}}{r} h_{\theta}^{I}+\frac{g^{I} h^{I}}{r}+\frac{1}{r} P_{\theta}^{I}=\frac{1}{\operatorname{Re}}\left[\nabla^{2} h^{I}-\frac{h^{I}}{r^{2}}+\frac{2}{r^{2}} g_{\theta}^{I}\right), \\
T_{1, t}+g^{I} T_{1, r}+\frac{h^{I}}{r} T_{1, \theta}-(\gamma-1) M_{o}^{2} P_{t}^{I}=\frac{1}{\operatorname{Re} P r} \nabla^{2} T_{1}+(\gamma-1) \frac{M_{o}^{2}}{\operatorname{Re}} \Phi^{I}+\tau F_{1}^{I} F_{2}^{I} e^{T_{1}}, \\
F_{j, t}^{I}+g^{I} F_{j, r}^{I}+\frac{h^{I}}{r} F_{j, \theta}^{I}=\frac{1}{\operatorname{Re} S c} \nabla^{2} F_{j}^{I}, j=1,2, \\
\Phi^{I}=\left(h_{r}^{I}-\frac{h^{I}}{r}\right)^{2}+u_{r}^{I^{2}}+\frac{1}{r^{2}} u_{\theta}^{I^{2}} .
\end{gathered}
$$


where we have chosen the Damkohler number $D a$ to be

$$
D a=\frac{e^{Z e}}{\tau \beta Z e} .
$$

This particular choice of the Damkohler number ensures that a distinguished limit exists, in that the reaction rate term is of the same order in a Zeldovich number expansion as the time derivative terms (see, e.g., Buckmaster and Ludford, 1982). This implies that we are looking at the fast chemistry limit and thus selects our chemical time scale.

We first see that the continuity and momentum equations (3.3b-e) are decoupled from the rest of the system and thus, will completely determine $u^{I}, g^{I}, h^{I}$ and $P^{I}$. At $t=0$, we situate a point vortex at the origin and allow it to diffuse under the action of viscosity. Then, assuming the flow in the $(r, \theta)$ plane to be axisymmetric, the induced non-dimensional flow field is given by (Lamb, 1932)

$$
\begin{gathered}
g^{I}=0, \quad h^{I}=\frac{1}{r}\left[1-\exp \left(-\operatorname{Re} r^{2} / 4 t\right)\right], \\
P^{I}(r, t)=-\int_{r}^{\infty} \frac{h^{I^{2}}(\bar{r}, t)}{\bar{r}} d \bar{r} \equiv-\frac{\operatorname{Re}}{4 t}\left\{\frac{2}{\eta^{2}}\left(1-2 e^{-\eta^{2} / 4}+e^{-\eta^{2} / 2}\right)+E i\left(\eta^{2} / 4\right)-E i\left(\eta^{2} / 2\right)\right\},
\end{gathered}
$$

where $\eta=r \sqrt{\operatorname{Re}} / \sqrt{t}$. The solution (3.5)-(3.6) is the solution for the incompressible Oseen vortex. The velocity scale $V_{v}$ has been chosen so that the corresponding potential vortex at $L_{v}$ (i.e., at $r=1$ ) is unity, resulting in the relationship

$$
\frac{\Gamma}{2 \pi L_{v} V_{v}}=1
$$

where $\Gamma$ is the circulation parameter of the vortex. This particular choice shows that

$$
\operatorname{Re}=\frac{L_{v} V_{v}}{v}=\frac{\Gamma}{2 \pi v}=R_{o}
$$

where $R_{o}$ is the usual vortex Reynolds number. The streamwise velocity component $u^{I}$ is found from the equation

$$
u_{t}^{I}+\frac{h^{I}}{r} u_{\theta}^{I}=\frac{1}{\operatorname{Re}} \nabla^{2} u^{I}
$$

which is to be solved subject to the initial and boundary conditions,

$$
u^{I}=k_{1}+k_{2} z \quad \text { at } t>0, r \rightarrow \infty, 0<\theta<2 \pi, \text { and } t=0, r>0,0<\theta<2 \pi \text {, }
$$

where $k_{1}$ is the nondimensional velocity at the centerline and $k_{2}$ is the local nondimensional shear, defined as the ratio of the time scale of the vortex to that of the shear. In this study we shall assume that $k_{2}=O(1)$ 
so that the two time scales are comparable. At this order the streamwise vortex influences the streamwise velocity component and hence the associated spanwise vorticity, but not vice versa.

We note here that equation (3.7) has previously been considered by several authors for non-reacting flows. Pearson and Abernathy (1984), in the context of streamwise vortical structures found in turbulent boundary layers, constructed solutions which were valid for all Reynolds numbers. Corcos (1988), in the context of large scale structures found in mixing layers, has recently given an asymptotic solution in the limit of large Reynolds number. Because these authors used different notations and because these solutions are needed later for understanding certain aspects of ignition, we give below a summary of these solutions for completeness.

To examine the time evolution of the streamwise Oseen vortex in a shear flow, we define

$$
u^{I}(r, \theta, t)=k_{1}+k_{2}[r \sin \theta+\hat{u}(r, \theta, t)]
$$

where $\hat{u}$ is the disturbed velocity component owing to the presence of the vortex. Upon substituting (3.9) into (3.7), $\hat{u}$ is found to satisfy the convection-diffusion equation

$$
\hat{u}_{t}+\frac{1}{r^{2}}\left[1-\exp \left(-\operatorname{Re} r^{2} / 4 t\right)\right]\left(r \cos \theta+\hat{u}_{\theta}\right)=\frac{1}{\operatorname{Re}} \nabla^{2} \hat{u},
$$

subject to homogeneous initial and boundary conditions. This equation was previously considered by Pearson and Abernathy (1984) (see their equation (14)), in the context of streamwise vortical structures found in turbulent boundary layers. They employed the similarity type variable $r^{2} / 4 t$ and defined (in our notation) $\hat{u}=\operatorname{Real}\left(r F(r) e^{i \theta}\right)$. This choice led to an equation for the complex function $F(r)$ which was solved numerically after special treatment of the regular singular point at the origin. A different approach for solving (3.10), which is perhaps more instructive, is to first transform using the similarity variable $\eta=r \sqrt{\operatorname{Re}} / \sqrt{t}$ and then introducing the definition

$$
\hat{u}=\frac{\sqrt{t}}{\sqrt{\operatorname{Re}}}\left[u_{e}(\eta) \cos \theta+u_{o}(\eta) \sin \theta\right]
$$

this yields the coupled second-order differential equations,

$$
\begin{gathered}
\frac{1}{2}\left(u_{e}-\eta u_{e}^{\prime}\right)+\frac{\operatorname{Re}}{\eta^{2}}\left[1-\exp \left(-\eta^{2} / 4\right)\right]\left(u_{o}+\eta\right)=u_{e}^{\prime \prime}+\frac{1}{\eta} u_{e}^{\prime}-\frac{1}{\eta^{2}} u_{e}, \\
\frac{1}{2}\left(u_{o}-\eta u_{o}^{\prime}\right)-\frac{\operatorname{Re}}{\eta^{2}}\left[1-\exp \left(-\eta^{2} / 4\right)\right] u_{e}=u_{o}^{\prime \prime}+\frac{1}{\eta} u_{o}^{\prime}-\frac{1}{\eta^{2}} u_{o},
\end{gathered}
$$

subject to the boundary conditions,

$$
u_{e}(0)=u_{o}(0)=u_{e}(\infty)=u_{o}(\infty)=0
$$


Primes denote ordinary differentiation with respect to the similarity variable $\eta, u_{e}$ is the amplitude of the even mode, and $u_{o}$ is the amplitude of the odd mode. The system (3.12) depends only on the Reynolds number Re.

The coupled system (3.12) for the odd and even components subject to (3.13) represents the influence of the Oseen vortex on the streamwise velocity and is solved numerically using a 2nd-order finite difference scheme with an appropriate stretching in $\eta$. The solution to this system for a range of Reynolds numbers is given in Figure 2. As the Reynolds number increases, the number of oscillations in each of the components $u_{e}$ and $u_{o}$ increases while the region of oscillation moves away from the origin, thus establishing a core region. The solution difference $u_{d}=\eta \sin \theta+u_{e} \cos \theta+u_{o} \sin \theta$, where $u_{d}=\sqrt{\operatorname{Re}}\left(u^{I}-k_{1}\right) /\left(k_{2} \sqrt{t}\right)$, is plotted in Figure 3 for $\theta= \pm \pi / 2$ and for various values of Re. From this figure the extent of the core region is seen to grow as Re increases, and this core, once established, grows like $\sqrt{t}$.

To investigate the solution described above for large times, let $t \rightarrow \infty$, so that $\eta \rightarrow 0$ for fixed $r$. Hence from (3.11) we have

$$
\begin{aligned}
\hat{u} & =\lim _{t \rightarrow \infty} \frac{\sqrt{t}}{\sqrt{\operatorname{Re}}}\left[u_{e}(\eta) \cos \theta+u_{o}(\eta) \sin \theta\right] \\
& =\lim _{\eta \rightarrow 0} \frac{r}{\eta}\left[u_{e}(\eta) \cos \theta+u_{o}(\eta) \sin \theta\right] \\
& =\lim _{\eta \rightarrow 0} \frac{1}{\eta}\left[u_{e}(\eta) y+u_{o}(\eta) z\right] \\
& =a_{1} y+b_{1} z
\end{aligned}
$$

where from (3.12) and (3.13) it is easy to show that the asymptotic behaviour as $\eta \rightarrow 0$ is

$$
u_{e}=a_{1} \eta+O\left(\eta^{3}\right), \quad u_{o}=b_{1} \eta+O\left(\eta^{3}\right)
$$

where $a_{1}$ and $b_{1}$ are found numerically and are functions of the Reynolds number Re. Substituting (3.14) into (3.9) yields

$$
u^{l}=k_{1}+k_{2}\left[a_{1} y+\left(1+b_{1}\right) z\right]
$$

Comparing (3.16) to the initial condition (3.8), the effect of the vortex is seen to be the establishment of a new equilibrium shear profile within the viscous core region. In addition, as $\operatorname{Re} \rightarrow \infty$ the coefficients $a_{1} \rightarrow 0, b_{1} \rightarrow-1$, yielding $u^{I} \rightarrow k_{1}$; i.e., the streamwise velocity component becomes constant within the core at large vortex Reynolds numbers.

The streamwise Oseen vortex induces additional spanwise vorticity. This is seen by considering the total vorticity defined by $\underline{\boldsymbol{\omega}}=\hat{e}_{x} \omega+\underline{\omega^{\prime}}$, where $\omega$ is the streamwise vorticity component and $\underline{\boldsymbol{\omega}}$ is associated with the spanwise vorticity

$$
\underline{\omega}^{\prime}=\hat{e}_{y} \frac{\partial u}{\partial z}-\hat{e}_{z} \frac{\partial u}{\partial y} .
$$


The magnitude of the spanwise vorticity, defined by $G=\left|\underline{\omega^{\prime}}\right|$, is a function of Re and, at leading order, has the value

$$
G_{0}=\left|k_{2}\right|\left[a_{1}^{2}+\left(1+b_{1}\right)^{2}\right)^{1 / 2},
$$

at the center of the core. Figure 4 is a plot of $G_{0}$ versus $\operatorname{Re}$ for $k_{2}=1$. Note that as $\operatorname{Re} \rightarrow \infty, G_{0} \rightarrow 0$ which implies that there are no gradients within the center of the core and thus the core is completely established. From this figure, the core is $95 \%$ established at $\mathrm{Re} \approx 50$ which is in agreement with Figure 3 where a well defined core is clearly visible at $\mathrm{Re}=100$.

Even though the solution can be determined numerically for any Reynolds number, it is constructive to consider the limit as $\operatorname{Re} \rightarrow \infty$. The leading-order solution in this limit is found by a direct asymptotic investigation of (3.7) subject to the initial and boundary conditions (3.8). In the limit $\operatorname{Re} \rightarrow \infty$, an outer inviscid layer exists and is governed by the inviscid version of (3.7),

$$
u_{t}^{O}+\frac{1}{r^{2}} u_{\theta}^{O}=0
$$

where $u^{O}$ denotes the streamwise velocity $u^{I}$ in this outer layer. This equation is valid for $r=O(1)$ and $t \ll O(R e)$, so that the effect of a potential line vortex or an Oseen vortex on the streamwise velocity is the same. The solution found by the method of characteristics is

$$
u^{o}=k_{1}+k_{2} r \sin \theta_{0}
$$

where $\theta_{0}$ is the characteristic

$$
\theta_{0}=\theta-\frac{t}{r^{2}}
$$

As $r \rightarrow 0$, the solution (3.20) is singular indicating that the viscous terms in (3.7) must be retained. We introduce the similarity type variable

$$
s=\frac{r}{\sqrt{\delta t}}
$$

where $\operatorname{Re}^{-1}<<\delta<1$ and $\delta$ is a nondimensional parameter that will be chosen in the course of the analysis. Defining $u^{M}$ to be the dependent variable $u^{I}$ in the main layer, and letting

$$
u^{M}=k_{1}+k_{2} \sqrt{\delta t} \tilde{u}(s, \theta)
$$

we see that $\tilde{u}$ satisfies the convection-diffusion equation

$$
1 / 2\left(\tilde{u}-s \tilde{u}_{s}\right)+\frac{1}{\delta s^{2}}\left[1-\exp \left(-\operatorname{Re} \delta s^{2} / 4\right)\right] \tilde{u}_{\theta}=\frac{1}{\delta \operatorname{Re}} \nabla^{2} \tilde{u} .
$$


To facilitate matching with the outer inviscid solution (3.20), we change to a new (non-orthogonal) coordinate system in which the characteristic $\theta_{0}$ is one of the coordinates. Thus, the above equation in terms of $s$ and $\theta_{0}$ becomes

$$
\begin{aligned}
1 / 2\left(\tilde{u}-s \tilde{u}_{s}\right) & -\frac{1}{\delta s^{2}} \exp \left(-\operatorname{Re} \delta s^{2} / 4\right) \tilde{u}_{\theta_{0}} \\
= & \frac{1}{\delta \operatorname{Re}}\left\{\left[\frac{\partial}{\partial s}+\frac{2}{\delta s^{3}} \frac{\partial}{\partial \theta_{0}}\right]^{2}+\frac{1}{s}\left[\frac{\partial}{\partial s}+\frac{2}{\delta s^{3}} \frac{\partial}{\partial \theta_{0}}\right]+\frac{1}{s^{2}} \frac{\partial^{2}}{\partial \theta_{0}^{2}}\right\}(\tilde{u}) .
\end{aligned}
$$

Balancing the viscous terms with the convection terms necessitates the choice

$$
1=\frac{1}{\delta \operatorname{Re}} \frac{4}{\delta^{2}}
$$

or, in terms of $\delta$,

$$
\delta=(4 / R e)^{1 / 3} \equiv O\left(v^{1 / 3}\right)
$$

which is in agreement with Corcos (1988). This layer is thicker than the inner diffusion layer of the vortex which is of the order $v^{1 / 2}$. For this scaling, $\exp \left(-\operatorname{Re} \delta s^{2} / 4\right)$ is exponentially small in the distinguished limit $\operatorname{Re}>>1$, and so again the effect of a potential line vortex or an Oseen vortex is the same. In order to account for the diffusion of the vortex, an inner layer must be constructed. This is not necessary, as will be shown below, since $u^{M}$ is not singular at the origin. The leading order equation in a $\delta$-expansion is given by

$$
1 / 2\left(\tilde{u}-s \tilde{u}_{s}\right)=\frac{1}{s^{6}} \tilde{u}_{\theta_{0} \theta_{0}},
$$

which is solved by separation of variables and matched with the outer solution (3.20) to get

$$
\tilde{u}=s \exp \left[\frac{-1}{3 s^{6}}\right] \sin \theta_{0}
$$

The solution in the main layer is now given by

$$
u^{M}=k_{1}+k_{2} s \sqrt{\delta t} \exp \left[\frac{-1}{3 s^{6}}\right] \sin \theta_{0}
$$

The composite solution, valid in both the outer and main layers, is given by

$$
u^{C}=k_{1}+k_{2} r \exp \left[\frac{-(\delta t)^{3}}{3 r^{6}}\right] \sin \left[\theta-\frac{t}{r^{2}}\right] \text {. }
$$


To compare the asymptotic solution $u^{C}$ given in (3.31) to the exact solution $u^{I}$ given in (3.9), a plot of the solution difference $u_{d}$ versus $\eta$ for $\theta= \pm \pi / 2$ with $\operatorname{Re}=10,000$ is shown in Figure 5, where $u_{d}$ and $\eta$ are defined as before. From this figure we see that the asymptotic solution is in good quantitative agreement with the exact solution shown in Figure $3 \mathrm{c}$.

The magnitude of the spanwise vorticity at $O(1)$ obtained by substituting (3.31) into the definition of $G$ and then letting $\delta \rightarrow 0$, yields

$$
G_{0}=\frac{2\left|k_{2}\right|}{\delta s^{2}} \exp \left[\frac{-1}{3 s^{6}}\right]\left|\cos \left(\theta-\frac{1}{\delta s^{2}}\right)\right|
$$

Figure 6 is a plot of $G_{0}$ versus $s$ for $\theta=\pi / 2, k_{2}=1$, and $\delta=1 / 5$. The limit $\operatorname{Re} \rightarrow 0$ corresponds to $G_{0} \rightarrow 0$, and so the large spikes have been created by the vortex.

With $g^{I}, h^{I}$ and $u^{I}$ now known, the following equations can be solved for $T_{1}$ and $F_{j}^{I}$

$$
\begin{gathered}
T_{1, t}+\frac{h^{I}}{r} T_{1, \theta}=\frac{1}{\operatorname{Re} P r} \nabla^{2} T_{1}+(\gamma-1) M_{o}^{2}\left[P_{t}^{I}+\frac{1}{\operatorname{Re}} \Phi^{I}\right]+\tau F_{1}^{I} F_{2}^{I} e^{T_{1},} \\
F_{j, t}^{I}+\frac{h^{I}}{r} F_{j, \theta}^{I}=\frac{1}{\operatorname{Re} S c} \nabla^{2} F_{j}^{I}, \quad j=1,2 .
\end{gathered}
$$

Note that the right hand side of the equation for the temperature is the sum of three terms: the diffusion term, the term due to compressible effects, and the reaction term, respectively. The appropriate boundary and initial conditions are given by

$$
\begin{aligned}
& T_{1}=0, F_{1}^{I}=1, F_{2}^{I}=0 \quad \text { at } t=0, r>0,0<\theta<\pi, \quad \text { and } t>0, r \rightarrow \infty, 0<\theta<\pi, \\
& T_{1}=\beta_{T}, F_{1}^{I}=0, F_{2}^{I}=\phi^{-1} \quad \text { at } t=0, r>0, \pi<\theta<2 \pi, \quad \text { and } t>0, r \rightarrow \infty, \pi<\theta<2 \pi,
\end{aligned}
$$

where $\phi$ is the equivalence ratio defined as the ratio of the initial mass fraction of the fuel $F_{1, \infty}$ to the initial mass fraction of the oxidizer $F_{2,-\infty}$, and $\beta_{T}$ is the parameter which allows for small initial temperature differences across the interface. If $\phi=1$, the mixture is said to be stoichiometric; if $\phi>1$ it is fuel rich; and if $\phi<1$, it is fuel lean. Also, if $\beta_{T}$ is less than zero, the oxidizer (species 2) is relatively cold compared to the fuel (species 1); and if $\beta_{T}$ is greater than zero, it is relatively hot. As $t$ increases, the solution for $T_{1}$ becomes unbounded at some finite time $\left(t_{i g}\right)$ and location $\left(y_{i g}, z_{i g}\right)$. This characterizes the ignition regime. Finally, with $T_{1}$ determined, the density perturbation $\rho_{1}$ can be found from (3.3a).

The case $M_{o}=0$ was discussed in the Introduction. Thus, the goal is to examine the influence of the compressible term in (3.33) on ignition. However, it is instructive to first investigate its influence on the temperature field in the absence of chemistry. We do this in the next section. The following section then contains numerical results when chemistry is included.

\subsection{Nonreacting Case}


In this section we investigate the influence of the compressible term in (3.33) on the temperature field in the absence of chemistry. Since an exact solution of (3.33) with $\tau=0$ is not possible, we shall examine the equation in the limit $\operatorname{Re}>>1$. In this limit an outer inviscid layer exists and is governed by the inviscid version of (3.33) for the outer variable $T_{1}^{O}$. Transforming to the $\left(r, \theta_{0}, t\right)$ characteristic coordinate system, where $\theta_{0}$ is given in (3.21), the temperature equation (3.33) becomes to leading order

$$
\begin{array}{r}
T_{1, t}^{O}=\frac{1}{\operatorname{Re} P r}\left\{\left[\frac{\partial}{\partial r}+\frac{2 t}{r^{3}} \frac{\partial}{\partial \theta_{0}}\right]^{2}+\frac{1}{r}\left[\frac{\partial}{\partial r}+\frac{2 t}{r^{3}} \frac{\partial}{\partial \theta_{0}}\right]+\frac{1}{r^{2}} \frac{\partial^{2}}{\partial \theta_{0}^{2}}\right\}\left(T_{1}^{O}\right) \\
+\frac{\gamma-1}{\operatorname{Re}} M_{o}^{2}\left[\frac{4}{r^{4}}+k_{2}^{2}\left\{\left[\tilde{u}_{r}+\frac{2 t}{r^{3}} \tilde{u}_{\theta_{0}}\right]^{2}+\frac{1}{r^{2}} \tilde{u}_{\theta_{0}}^{2}\right\}\right]+O\left(R e^{-2}\right),
\end{array}
$$

where $\tilde{u}=r \sin \theta_{0}$ and we have made use of the leading order outer solutions $h^{I}=1 / r+e s t$ and $P^{I}=-1 /\left(2 r^{2}\right)+$ est, where est means exponentially small terms in Re, valid for $t<<O(R e)$. The leading order solution in a large Reynolds number expansion can be shown to be

$$
T_{1}^{o}=\frac{\gamma-1}{\operatorname{Re}} M_{o}^{2}\left\{\frac{4 t}{r^{4}}+k_{2}^{2}\left[t+\frac{t^{2}}{r^{2}} \sin \left(2 \theta_{0}\right)+\frac{4 t^{3}}{3 r^{4}} \cos ^{2} \theta_{0}\right]\right\}+O\left(R e^{-2}\right) .
$$

Note that the terms in the brackets are associated with the streamwise vortex and with the spanwise vorticity. In the absence of the spanwise vorticity (i.e., $k_{2}=0$ ), the first term is positive showing that the effect of compressibility is to heat the outer region of the viscous core. With $T_{1}^{O}$ known, the density in the outer layer can be determined from (3.3a).

As $r \rightarrow 0$, the solution (3.37) becomes singular so that the viscous terms in (3.33) must be retained. Transforming to the $\left(s, \theta_{0}, t\right)$ coordinate system, where $s$ is defined in (3.22) and $\theta_{0}=\theta-1 / \delta s^{2}$, the temperature equation (3.33) in the main layer becomes

$$
\begin{gathered}
T_{1, t}^{M}-\frac{s}{2 t} T_{1, s}^{M}=\frac{1}{\delta \operatorname{Re} P r t}\left\{\left[\frac{\partial}{\partial s}+\frac{2}{\delta s^{3}} \frac{\partial}{\partial \theta_{0}}\right]^{2}+\frac{1}{s}\left[\frac{\partial}{\partial s}+\frac{2}{\delta s^{3}} \frac{\partial}{\partial \theta_{0}}\right]+\frac{1}{s^{2}} \frac{\partial^{2}}{\partial \theta_{0}^{2}}\right\}\left(T_{1}^{M}\right) \\
+\frac{\gamma-1}{\operatorname{Re}} M_{0}^{2}\left[\frac{4}{\delta^{2} t^{2} s^{4}}+k_{2}^{2}\left\{\left[\tilde{u}_{s}+\frac{2}{\delta s^{3}} \tilde{u}_{\theta_{0}}\right]^{2}+\frac{1}{s^{2}} \tilde{u}_{\theta_{0}}^{2}\right\}\right],
\end{gathered}
$$

where, to leading order in $\delta$,

$$
\tilde{u}=s \exp \left[\frac{-1}{3 s^{6}}\right] \sin \theta_{0}+O(\delta)
$$

To determine the proper expansion in the main layer and the appropriate matching condition as $s \rightarrow \infty$, we first expand the outer solution (3.37) as $r \rightarrow 0$, yielding

$$
T_{1}^{O} \approx(\gamma-1) M_{o}^{2}\left\{\frac{\delta}{t s^{4}}\left[1+\frac{k_{2}^{2} t^{2}}{3} \cos ^{2} \theta_{0}\right]+\frac{\delta^{2} k_{2}^{2} t}{4 s^{2}} \sin \left(2 \theta_{0}\right)+\frac{\delta^{3} k_{2}^{2} t}{4}\right\}+\cdots
$$


We now expand $T_{1}^{M}$ as

$$
T_{1}^{M}=\delta f_{1}+\delta^{2} f_{2}+\cdots
$$

as $\delta \rightarrow 0$. Substituting the above expansion into (3.38) gives the following problem at leading order:

$$
f_{1_{t}}-\frac{s}{2 t} f_{1_{s}}=\frac{1}{\operatorname{Pr} t s^{6}} f_{1_{\theta_{0} \theta_{0}}}+(\gamma-1) M_{o}^{2}\left\{\frac{1}{t^{2} s^{4}}+\frac{k_{2}^{2}}{s^{4}} \exp \left[\frac{-2}{3 s^{6}}\right] \cos ^{2} \theta_{0}\right\} .
$$

The appropriate solution that matches with the outer solution (3.40) can be verified to be

$$
\begin{gathered}
f_{1}=\frac{(\gamma-1) M_{o}^{2}}{t s^{4}}+\frac{\gamma-1}{4} M_{o}^{2} k_{2}^{2} t f_{11}\left(s, \theta_{0}\right), \\
f_{11}=s^{2}\left\{1-\exp \left[\frac{-2}{3 s^{6}}\right]+\frac{P r}{(2-P r)}\left\{\exp \left[\frac{-2}{3 s^{6}}\right]-\exp \left[\frac{-4}{3 P r s^{6}}\right]\right\} \cos \left(2 \theta_{0}\right)\right\},
\end{gathered}
$$

which decays as $s \rightarrow \infty$. The first term of $f_{1}$ is associated with the streamwise vortex while $f_{11}$ is associated with the spanwise vorticity. As mentioned above, the leading order term $f_{1}$ is positive showing that the effect of compressibility is to heat the outer region of the viscous core. Since we are primarily interested in the influence of the spanwise vorticity, we plot in Figure 7 a graph of $f_{11}$ as a function of $s$ for $\operatorname{Pr}=1, \theta=\pi / 2$, and (a) $\delta=1 / 5$ and (b) $\delta=1 / 8$. Note that the graph decays exponentially as $s \rightarrow \infty$, quadratically as $s \rightarrow 0$, and is positive and oscillates for $s \approx O(1)$. Also, the number of oscillations increases as $\delta$ decreases but stays within some temperature envelope. Thus the effect of the streamwise vortex is to introduce positive temperature fluctuations in the main layer which are damped out in the inner viscous layer. This result will have important implications in the ignition regime when chemistry is included, and will be brought out in the next section. The density can now be determined from (3.3a), and is given by

$$
\rho_{1}^{M}=-\frac{\gamma M_{o}^{2}}{2 s^{2} t \delta}-\delta f_{1}+\cdots
$$

As $s \rightarrow 0, T_{1}^{M}$ and $\rho_{1}^{M}$ become singular suggesting that an inner viscous layer is present. To determine the proper scalings, we see from the expansion (3.44) for the density that the second order term is of the same order as the leading order term when $s \rightarrow 0$, yielding the balance $s=O\left(\operatorname{Re}^{-1 / 3}\right)$. This suggests the new variable for the inner layer

$$
r=\frac{\xi}{\sqrt{\mathrm{Re}}}
$$

which is the size of the viscous core. Expanding the temperature and density in the main layer in terms of the inner variable and taking the limit $\operatorname{Re} \rightarrow \infty$, the proper expansion for the inner variables are

$$
T_{1}^{I}=\operatorname{Re} \hat{T}, \quad \rho_{1}^{I}=\operatorname{Re} \hat{\rho},
$$


where the superscript indicates variables in the inner layer. The leading order equations in a Reynolds number expansion are given by

$$
\begin{gathered}
\hat{\rho}+\hat{T}=\gamma M_{o}^{2} \hat{P} \\
\hat{T}_{t}-(\gamma-1) M_{o}^{2} \hat{P}_{0_{t}}=\frac{1}{P r}\left(\hat{T}_{\xi \xi}+\frac{\hat{T}_{\xi}}{\xi}\right)+(\gamma-1) M_{o}^{2}\left(\hat{h}_{0_{\xi}}-\frac{\hat{h}_{0}}{\xi}\right)^{2},
\end{gathered}
$$

where

$$
h_{0}=\operatorname{Re}^{1 / 2} \hat{h}_{0}(\xi, t), \quad P_{0}=\operatorname{Re} \hat{P}_{0}(\xi, t)
$$

$\hat{h}_{0}$ and $\hat{P}_{0}$ can be found from (3.5) and (3.6). This system is axisymmetric and is to be solved subject to appropriate matching conditions as $\xi \rightarrow \infty$. We do not solve this system here, but rather note that the variables are bounded at the origin. Furthermore, this system is equivalent to that considered by Colonius et al. (1991) if we rewrite their system in our notation, and so we refer the interested reader to that paper for further details.

\subsection{Reacting Case}

When chemical effects are included, the system (3.33)-(3.34) with boundary conditions (3.35) describes the ignition regime which must be solved numerically. The numerical scheme utilized here is described in Macaraeg et al. (1992). A small nonzero initial time is assumed in order to avoid nonphysical initial transients which may lead to early ignition. For the cases which follow, $k_{1}$ is assumed to be zero, so that the imposed shear is symmetric about the origin. Emphasis will be placed on the effects of the shear strength parameter $k_{2}$, so the other parameters will be held fixed. Here, we set $\beta_{T}=0$ and $\phi=\operatorname{Pr}=1$.

We begin the numerical computations by first setting $\tau=0$ in (3.33). This allows a qualitative comparison between the numerically generated solution and the asymptotic solution derived in the previous section. To this end, recall that the asymptotic solution indicates that the introduction of streamwise shear gives rise to oscillations in the velocity field as well as the temperature field via the dissipation function. Shown in Figure 8 are contour plots and cross-section cuts of $T_{1}$ and $T U$ for $\operatorname{Re}=50, k_{2}=1$ and $M_{o}=0.7$. The quantity $T U$ is computed by subtracting the numerical solution corresponding to $M_{0}=0$ from $T_{1}$. Thus, $T U$ is proportional to $f_{11}$ of the previous section and highlights the underlying oscillating temperature field. The cross-section plots of $T_{1}$ and $T U$ versus $z$, taken through the vortex center, show that the overall temperature perturbation has a single temperature peak in the center, whereas the temperature due to spanwise vorticity has multiple peaks, even though their magnitude is small at this low shear strength. If the strength of the shear is increased, the magnitude of these oscillations increases by $k_{2}^{2}$, as can be seen in Figure 9 which displays plots of $T U$ for three values of the shear strength parameter $k_{2}$. This trend was predicted earlier by the outer asymptotic solution obtained in (3.43), which indicates a proportionality with $k_{2}^{2}$. Also given are the corresponding cross-sections of the streamwise velocity component. Note that the magnitude and number of oscillations increases with increasing shear, again consistent with the asymptotic solution derived in the previous section. Figure 10 depicts contours and cross-sections of $T_{1}$ for the same conditions of Figure 9. Note that the temperature perturbation, $T_{1}$, becomes dominated by the spanwise vorticity for high values of shear. 
The multiple temperature peaks appearing for high shear values can have a dramatic effect on ignition when chemistry is added. To illustrate this we show in Figure 11 contours and cross-section plots of the temperature perturbation for $\mathrm{Re}=10, M_{o}=0.316$ and two values of the shear strength parameter $k_{2}$. For $k_{2}=1$, there is a single hot spot located in the vortex center, while for high shear $\left(k_{2}=20\right)$ there are two ignition points, located in the main layer of the viscous core. The evolution in time of this double ignition point is shown in Figure 12. Note that the temperature perturbation is strongly sheared, which gives rise to multiple ignition points at a later time.

The influence of the streamwise shear on the ignition time is shown in Figure 13, where the maximum temperature perturbation over the entire spatial domain is plotted against time. We note here that for weak shear $\left(k_{2}=1\right)$, the ignition time is comparable to that obtained by Linan and Crespo (1976) in which diffusion is the only mechanism present for mixing. Also, the ignition location is at the center of the vortex, consistent with previous results (Macaraeg et al., 1992). As the strength of the shear increases, the ignition time decreases indicating that mixing is enhanced owing to the presence of streamwise shear. In addition, the ignition location no longer resides in the viscous core, but now multiple ignition points occur and are located in the outer region of the core. This phenomena is due to the large temperature perturbations induced by the interaction process between the streamwise and spanwise vorticity described in the previous section.

\section{CONCLUSIONS}

The problem of a flame interacting with a vortex is assumed to model certain fundamental mechanisms of turbulent diffusion flames which form the basis of many propulsion devices. Streamwise shear, which can be present due to large scale structures within the flow field or can be introduced as a mixing enhancement technique, is always present in these combustion systems, so that it is important to study the role of shear in such flame/vortex interactions. An unexplored fundamental problem of turbulent reacting flows is thus the evolution of a shear flow in the presence of streamwise vortices, or conversely, the dynamics of vortices stretched by shear flow. This problem is examined by means of asymptotic analysis and numerical simulation in the limit of small Mach number. An idealized model is employed to describe the interaction process which, among other assumptions, neglects spatial variations in the streamwise direction. The model then consists of a one-step, irreversible Arrhenuis reaction between initially unmixed species occupying adjacent half-planes which are then allowed to mix and react in the presence of a streamwise vortex embedded in a shear flow.

It is shown that the effect of shear is to give rise to oscillations in the velocity field, which produces localized regions of high temperature gradients via viscous heating. As the strength of the shear is increased, these high temperature gradients give rise to multiple ignition points while substantially decreasing the overall ignition times. This results in the enhancement of mixing brought about by the linear shear associated with the spanwise vorticity. 


\section{REFERENCES}

Ashurst, W.T. \& Meiburg, E. (1988) Three-dimensional Shear Layers via Vortex Dynamics. J. Fluid Mech., 189, pp. 87.

Blackwelder, R.F. \& Eckelmann, H. (1979) Streamwise Vortices Associated with the Bursting Phenomenon. J. Fluid Mech., 94, pp. 577-594.

Buckmaster, J.D. and Ludford, G.S.S. (1982) Theory of Laminar Flames. Cambridge University Press, Cambridge.

Colonius, T., Lele, S.K. and Moin, P. (1991) The Free Compressible Viscous Vortex. Journal of Fluid Mechanics, 230, pp. 45-73.

Corcos, G.M. (1988) The Role of Cartoons in Turbulence. In Perspectives in Fluid Mechanics, ed. D. Coles, Lecture Notes in Physics, 320, Springer- Verlag, pp. 48-65.

Drummond, J.P. and Mukunda, H.S. (1988) A Numerical Study of Mixing Enhancement in Supersonic Reacting Flow Fields. AIAA Paper No. AIAA-88-3260.

Glassman, I. (1977) Combustion. Academic Press, Inc.

Jackson, T.L, Macaraeg, M.G. and Hussaini, M.Y. (1993) The Role of Acoustics in Flame/Vortex Interactions. Journal of Fluid Mechanics, 254, pp. 579-603.

Klebanoff, P.S., Tidstrom, K.D. \& Sargent, L.M. (1962) The Three-dimensional Nature of Boundary Layer Instability. J. Fluid Mech., 12, pp. 1-34.

Kozusko, F., Macaraeg, M.G. and Jackson, T.L. (1994) Study of Ignition of a Laminar Diffusion Flame in the Field of a Vortex Pair. in Transition, Turbulence and Combustion, T.B. Gatski, M.Y. Hussaini, T.L. Jackson, eds., Kluwer Publishing Co., in press.

Lamb, H. (1932) Hydrodynamics. Sixth edition, ed. Cambridge University Press, Cambridge.

Lasheras, J.C. \& Choi, H. (1988) Three-Dimensional Instability of a Plane Free Shear Layer: An Experimental Study of the Formation and Evolution of Streamwise Vortices. J. Fluid Mech., 189, pp. 53-86.

Lin, S.J. \& Corcos, G.M. (1984) The Mixing Layer: Determinstic Models of a Turbulent Flow. Part 3. The Effect of a Plane Strain on the Dynamics of Streamwise Vortices. J. Fluid Mech., 141, pp. 139-178.

Macaraeg, M.G., Jackson, T.L. and Hussaini, M.Y. (1992) Ignition and Structure of a Laminar Diffusion Flame in the Field of a Vortex. Combustion Science and Technology, 87, pp. 363-387.

Marble, F.E. (1985) Growth of a Diffusion Flame in the Field of a Vortex. In Recent Advances in Aerospace Sciences, ed. C. Cassci, Plenum, pp. 395-413.

Metcalfe, R.W., Orszag, S.A., Brachet, M.E., Menon, S. \& Riley, J.J. (1987) Secondary Instability of a Temporally Growing Mixing Layer. J. Fluid Mech., 184, pp. 207-243. 
Pearson, C.F. and Abernathy, F.H. (1984) Evolution of a Flow Field Associated with a Streamwise Diffusing Vortex. Journal of Fluid Mechanics, 146, pp. 271-283.

Willmarth, W.W. (1975) Structure of Turbulence in Boundary Layers. Adv. Appl. Mech., 15, pp. 159-254.

Wray, A.A. \& Hussaini, M.Y. (1984) Numerical Experiments in Boundary-Layer Stability. Proc. Roy. Soc. London A, 392, pp. 373-389.

Zang, T.A. \& Hussaini, M.Y. (1987) Numerical Simulation of Nonlinear Interactions in Channel and Boundary-Layer Transition. In Nonlinear Wave Interactions in Fluids, Miksad, R.W., Akylas, T.R. and Herbert, T., eds., AMD-Vol. 87, pp. 131-145. 


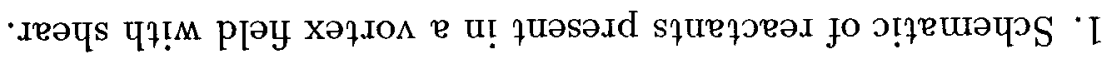

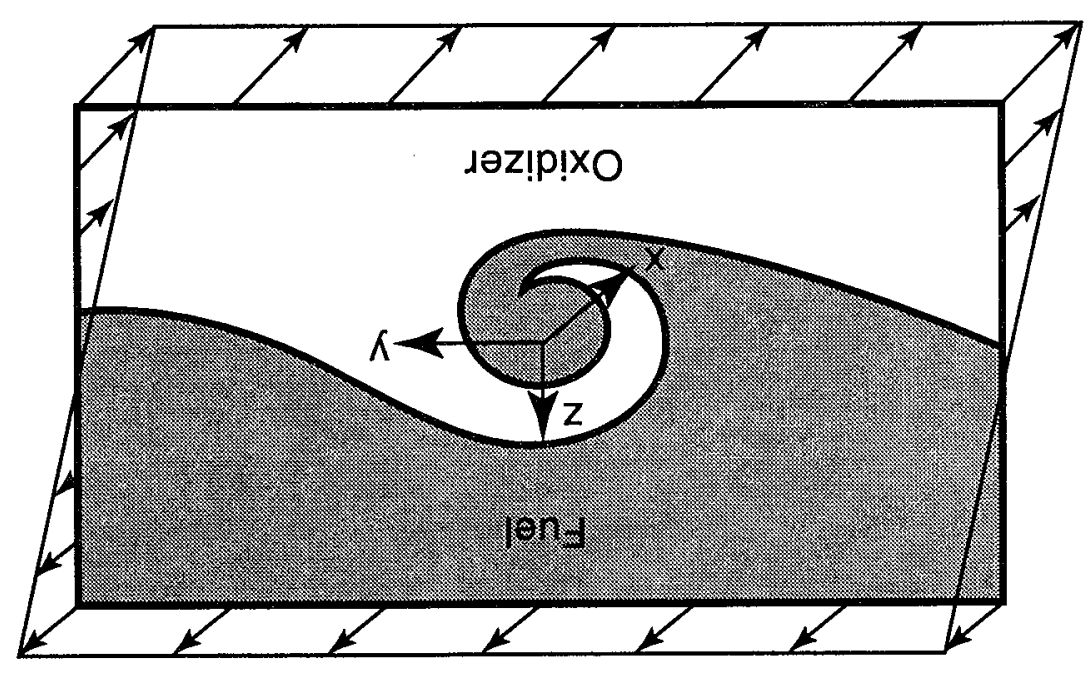



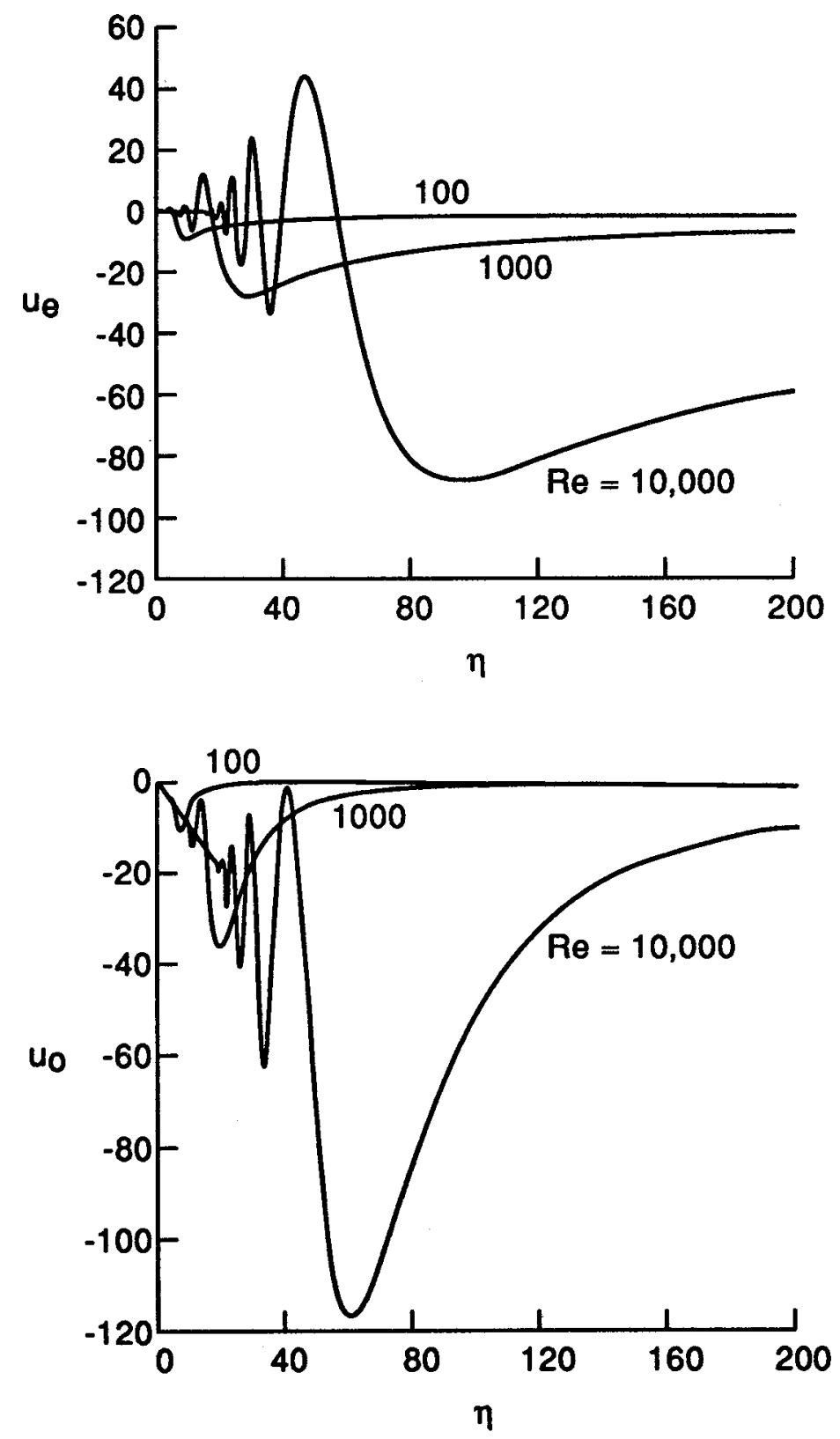

2. Plot of (a) $u_{e}$ versus $\eta$ for various values of the Reynolds number Re. 

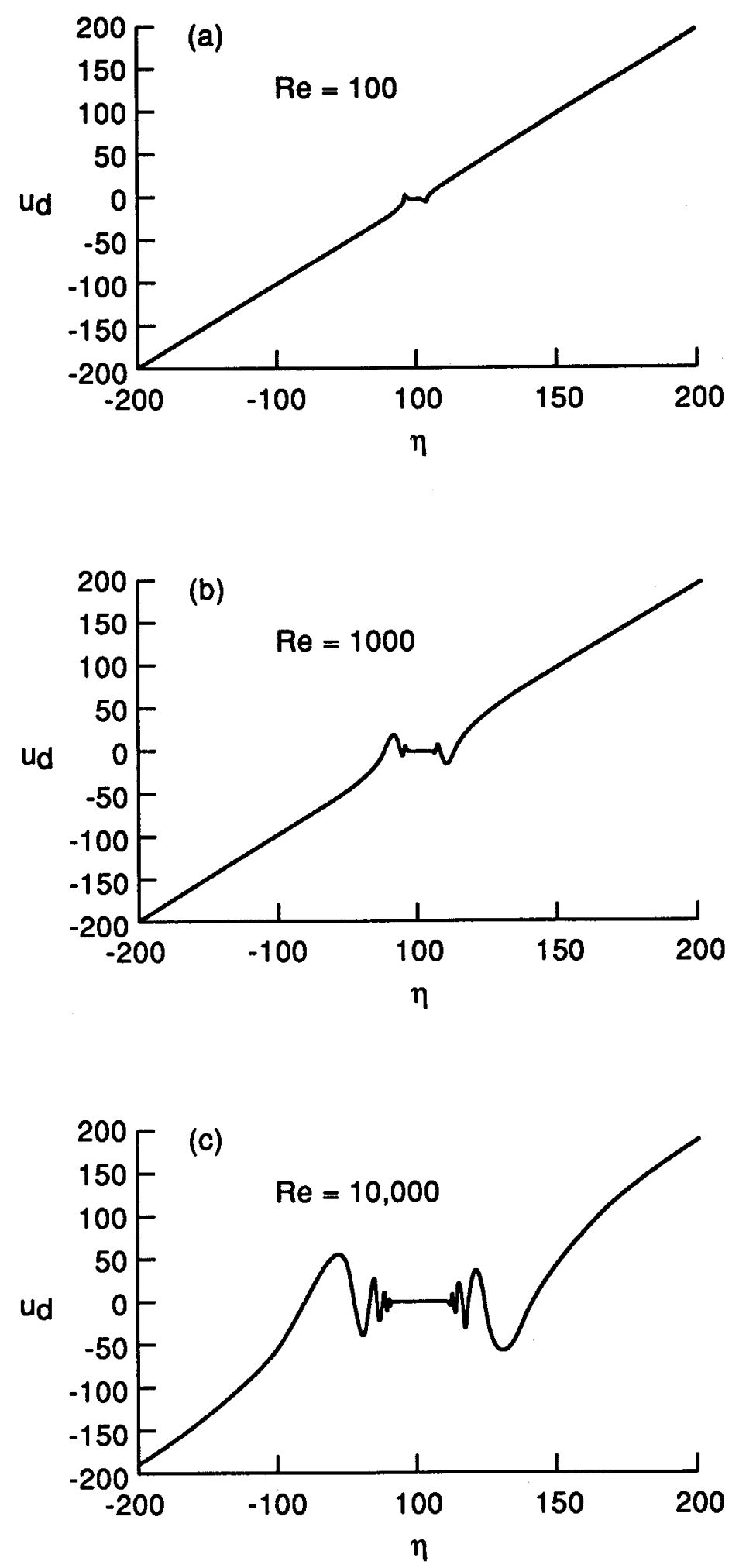

3. Plot of the solution difference $u_{d}$ versus $\eta$ for $\Theta= \pm \pi / 2$ and various values of the Reynolds number Re. 


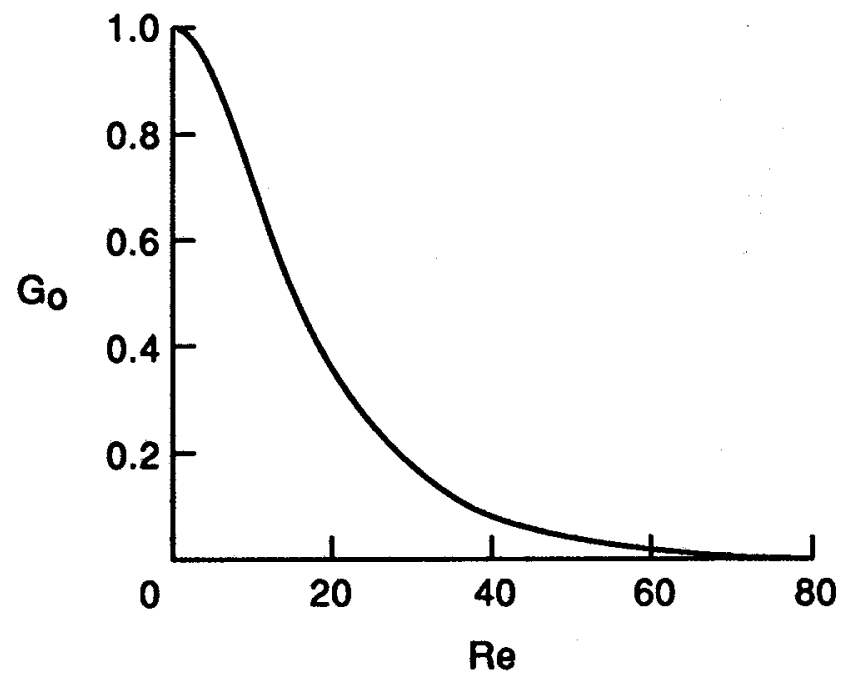

4. Plot of $G_{0}$ versus the Reynolds number Re for $k_{2}=1$. 


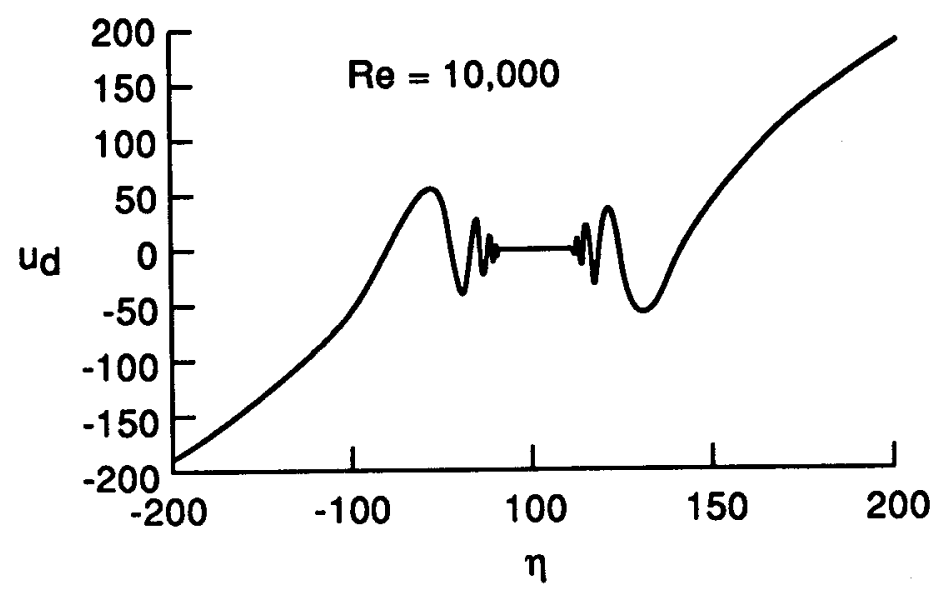

5. Plot of the solution difference $u_{d}$ versus $\eta$ for $\Theta= \pm \pi / 2$ and $\operatorname{Re}=10,000$. 

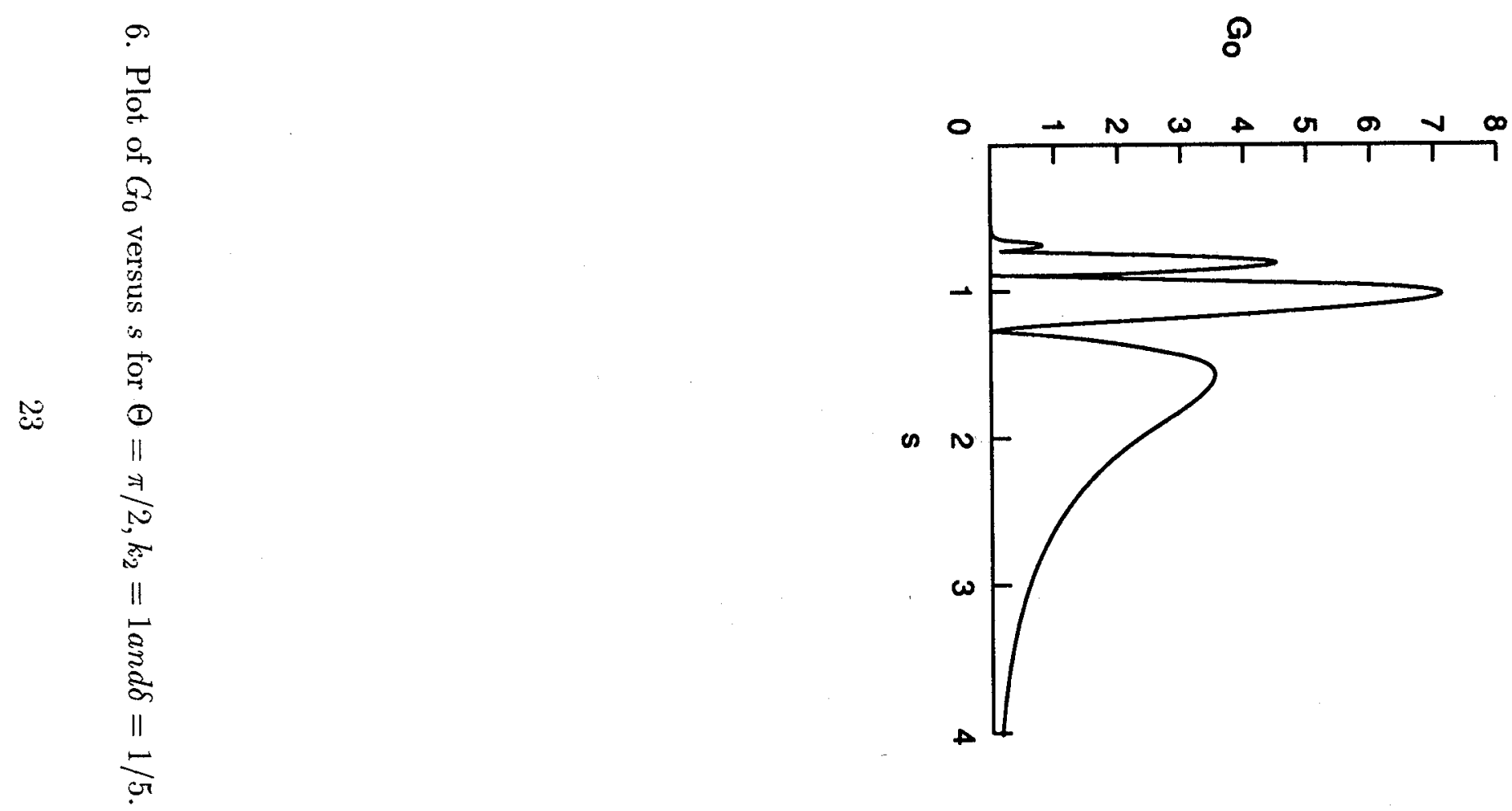

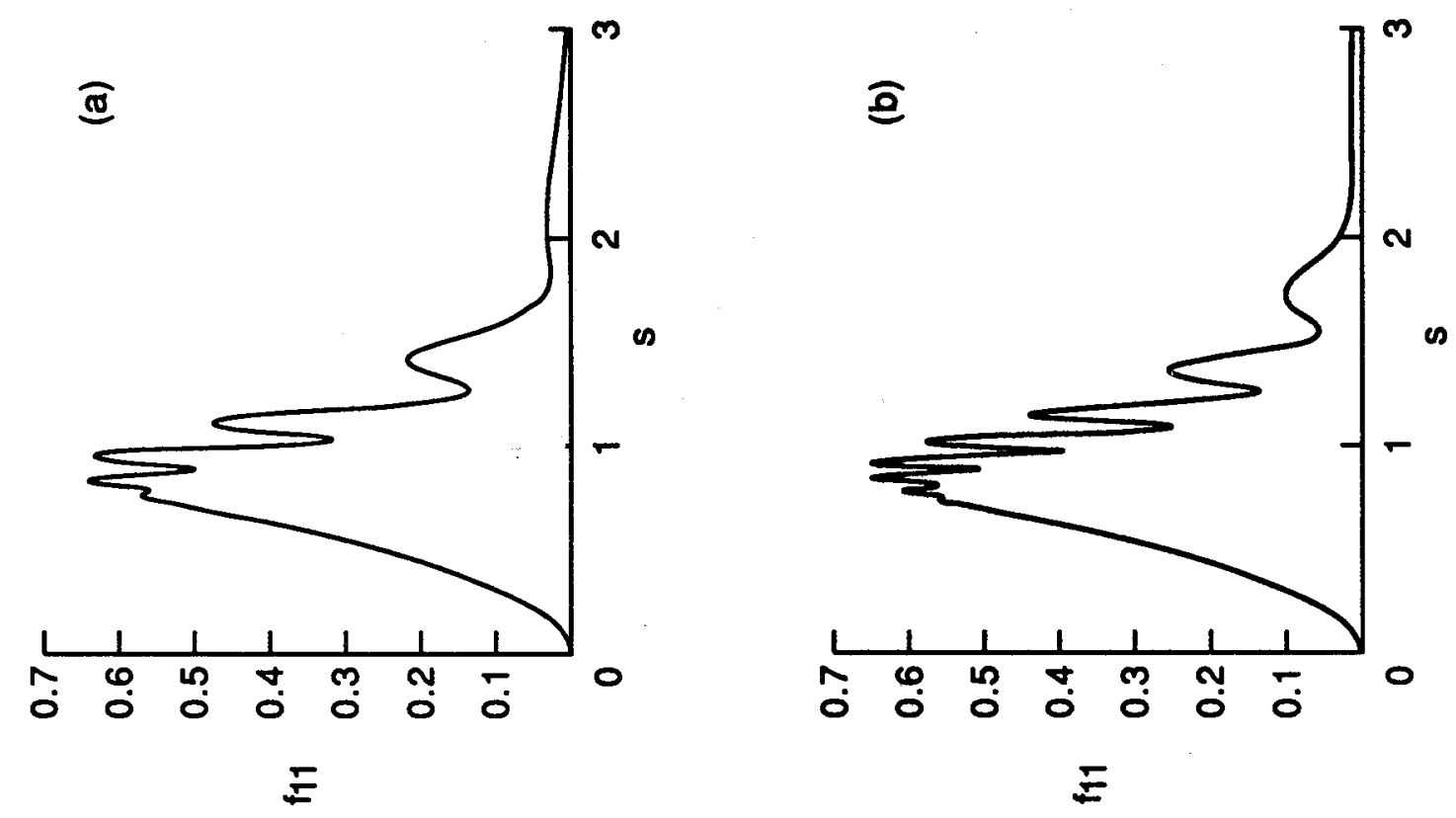


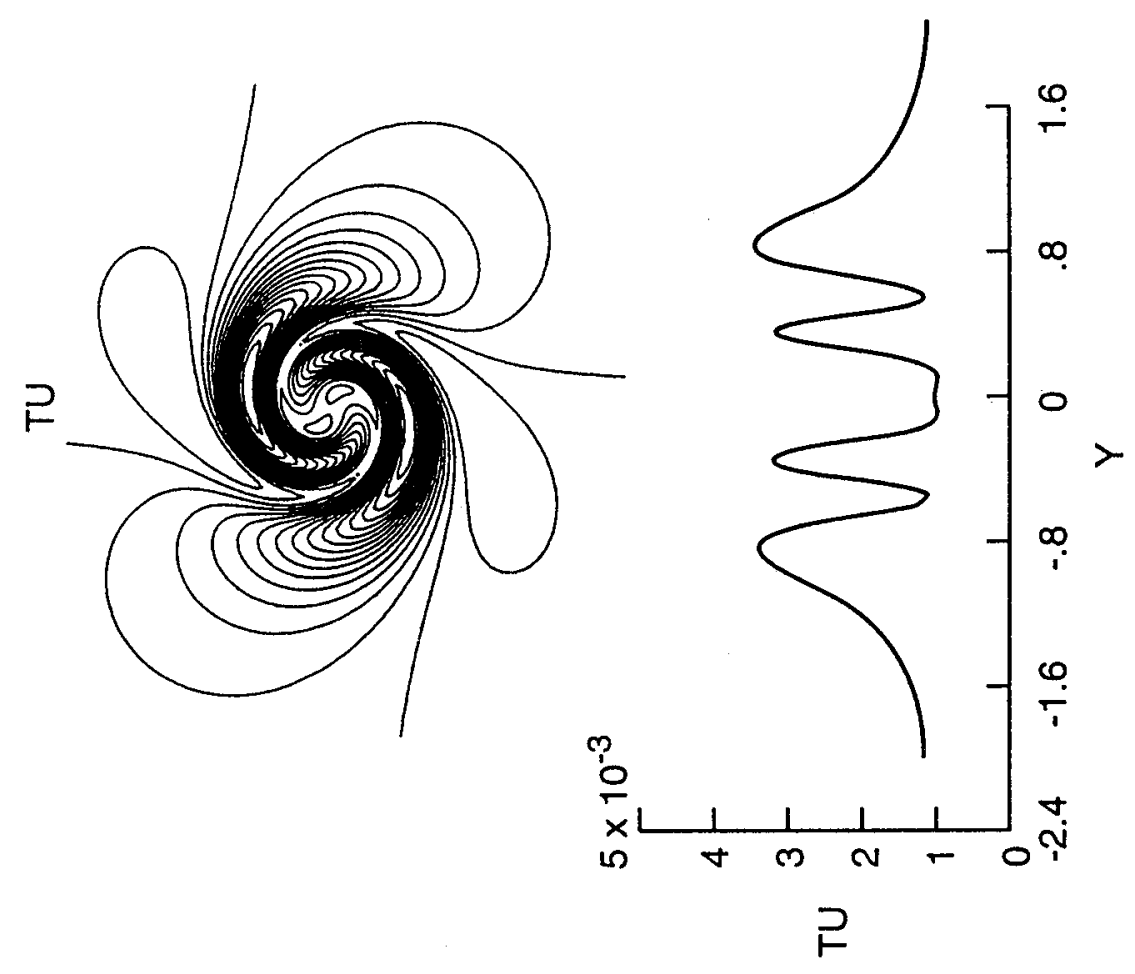

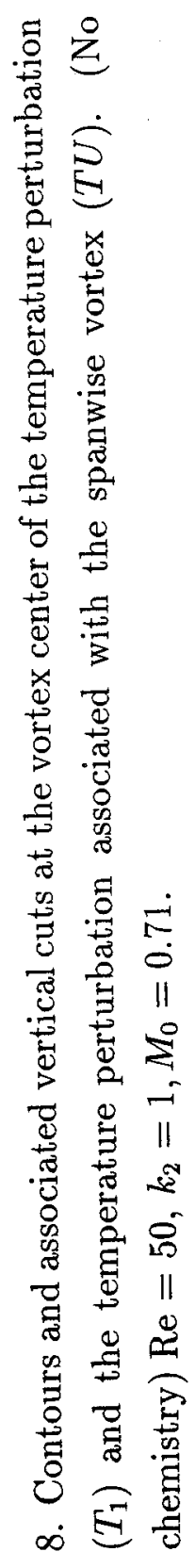

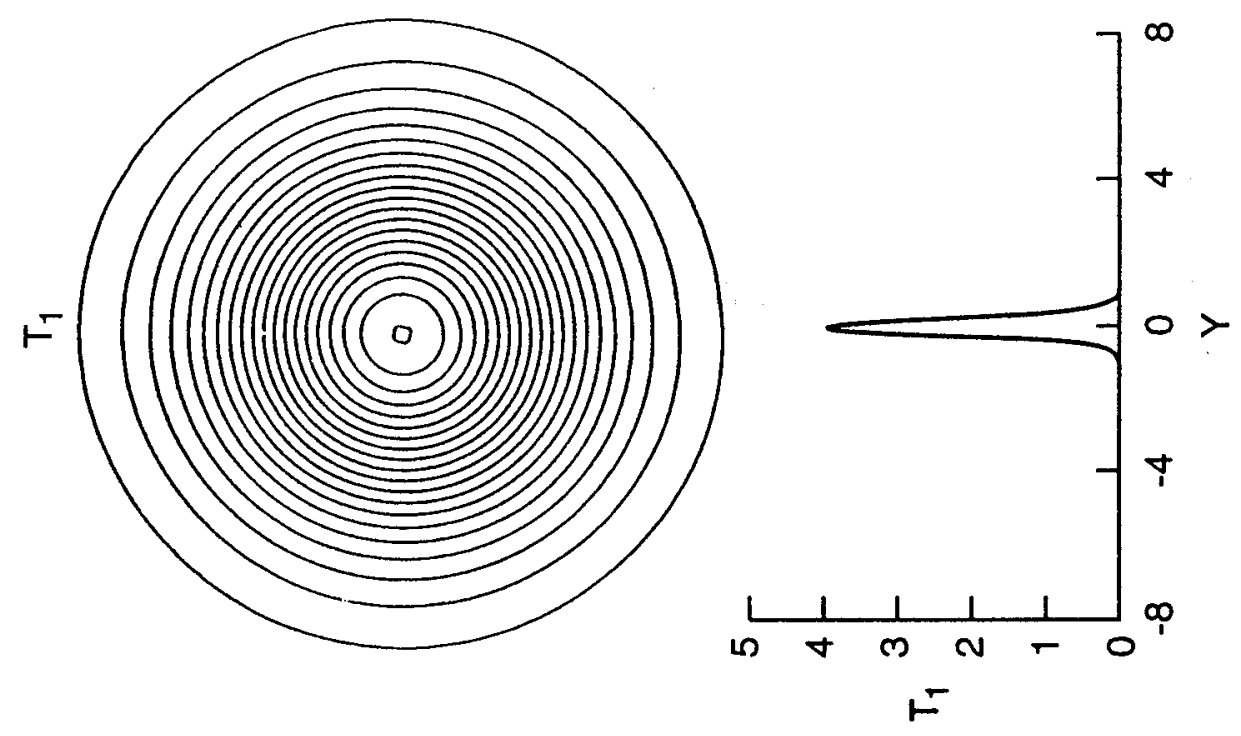



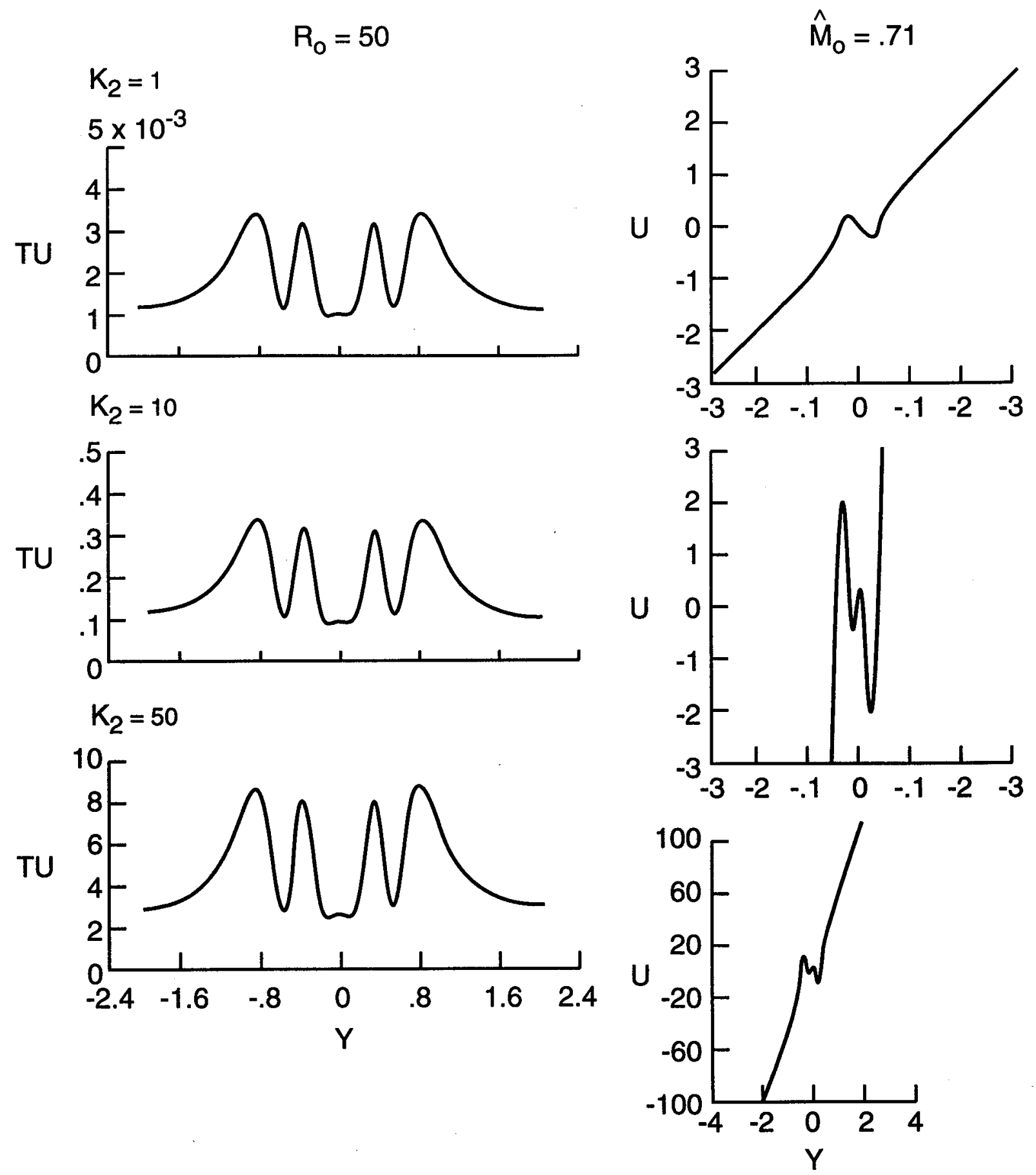

9. Vertical cuts at the vortex center of temperature perturbation due to the spanwise vorticity $(T U)$ and streamwise velocity $(U)$, for varying shear strengths. (No chemistry) $\operatorname{Re}=50$, $M_{0}=0.71$. 

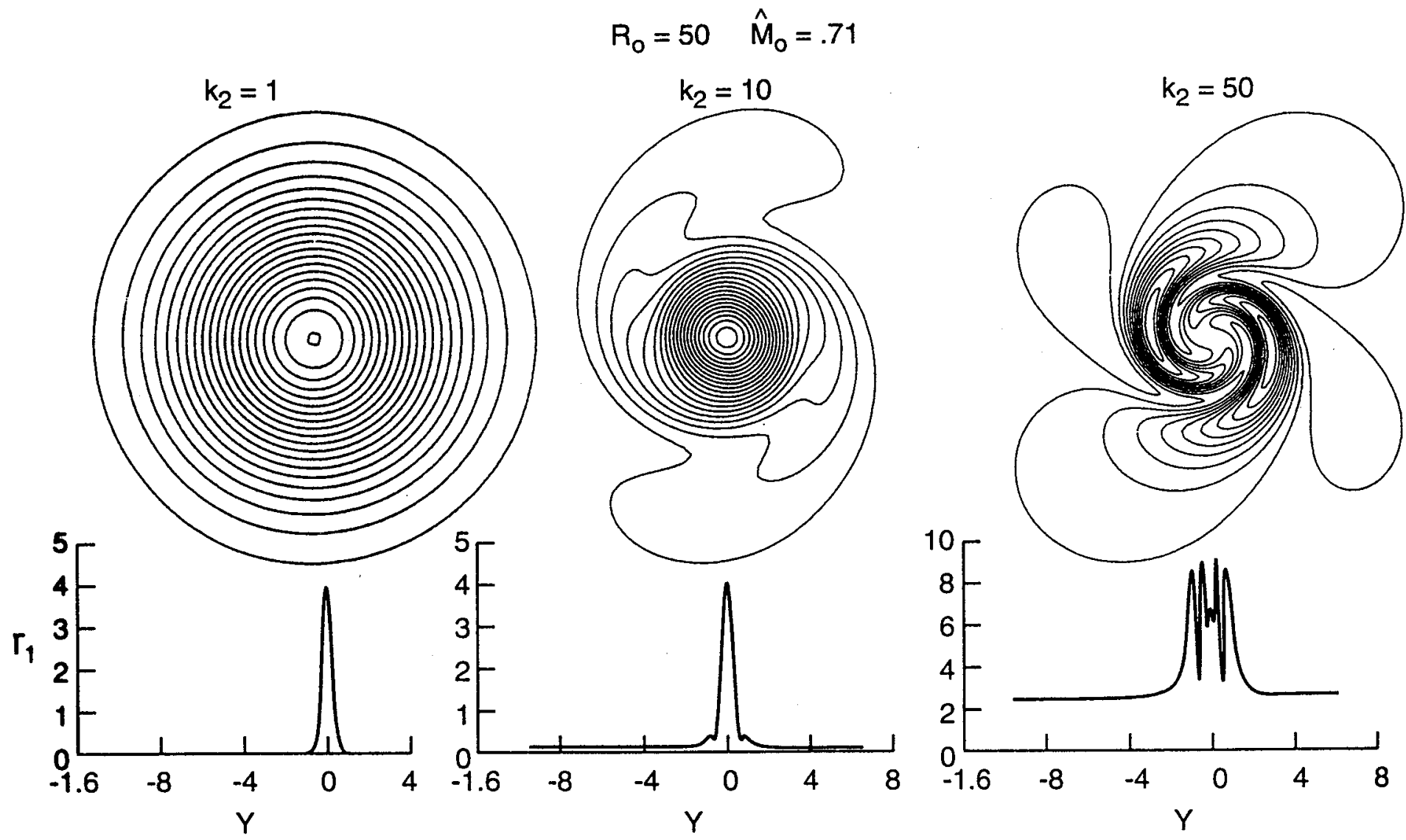

10. Contours and vertical cuts at the vortex center of the temperature perturbation $\left(T_{1}\right)$ for increasing shear strengths. (No chemistry) $T_{1}=50, M_{0}=0.71$. 


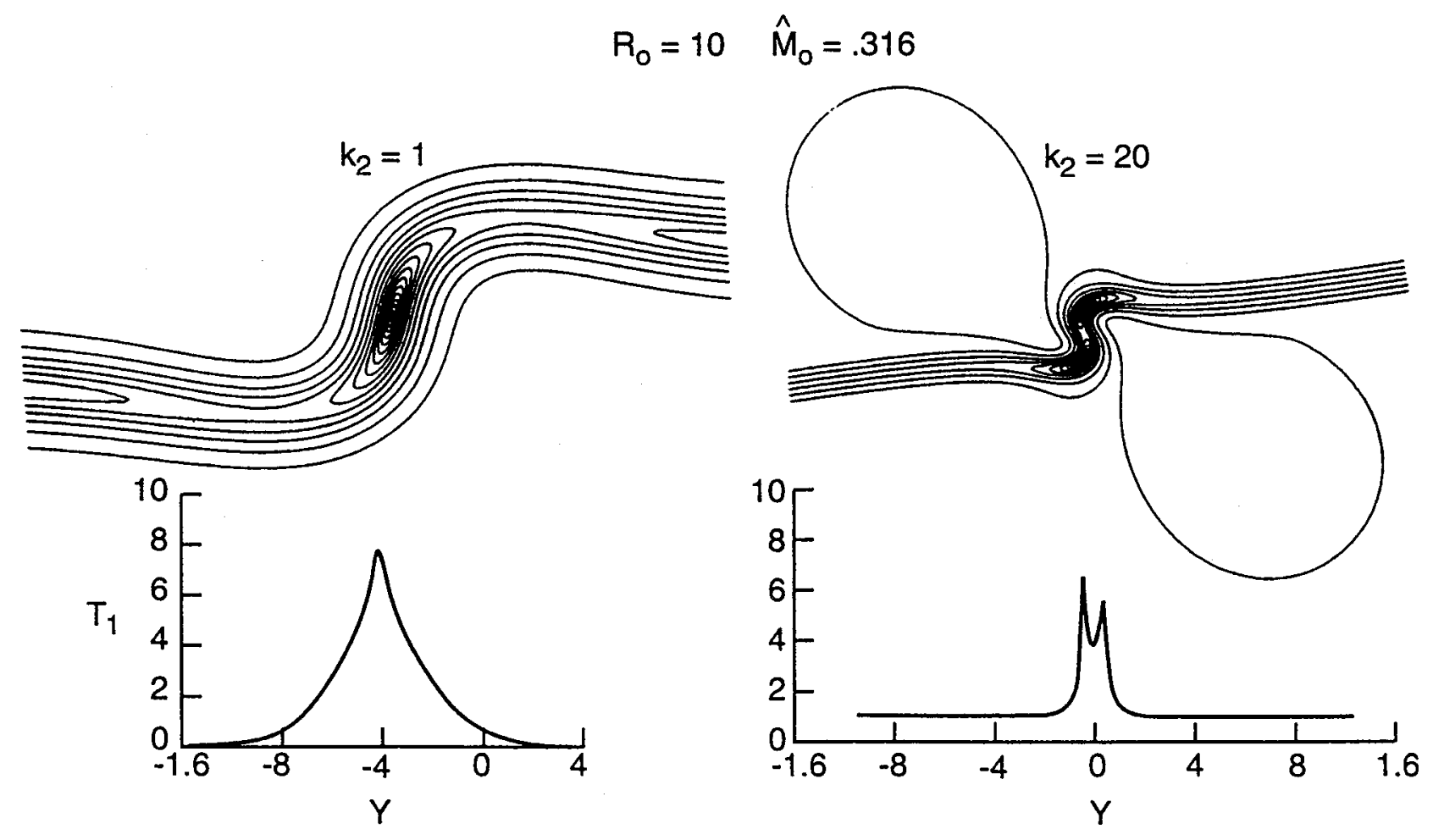

11. Contours and vertical cuts of the temperature perturbation $\left(T_{1}\right)$ at ignition for increasing values of shear. $\operatorname{Re}=10, M_{0}=0.316$. 


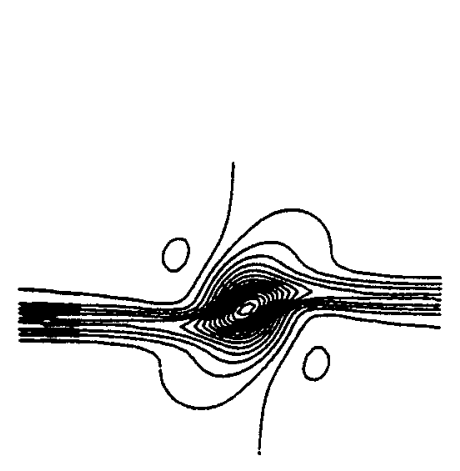

Time $=.25$

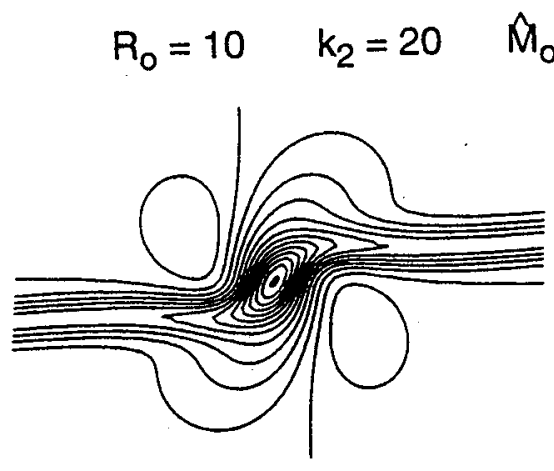

.5

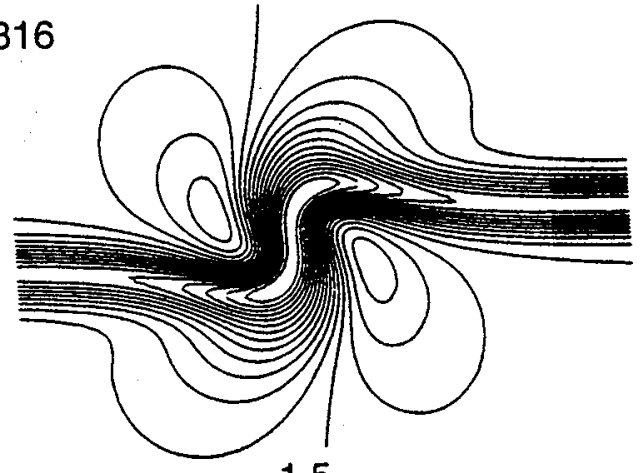

1.5
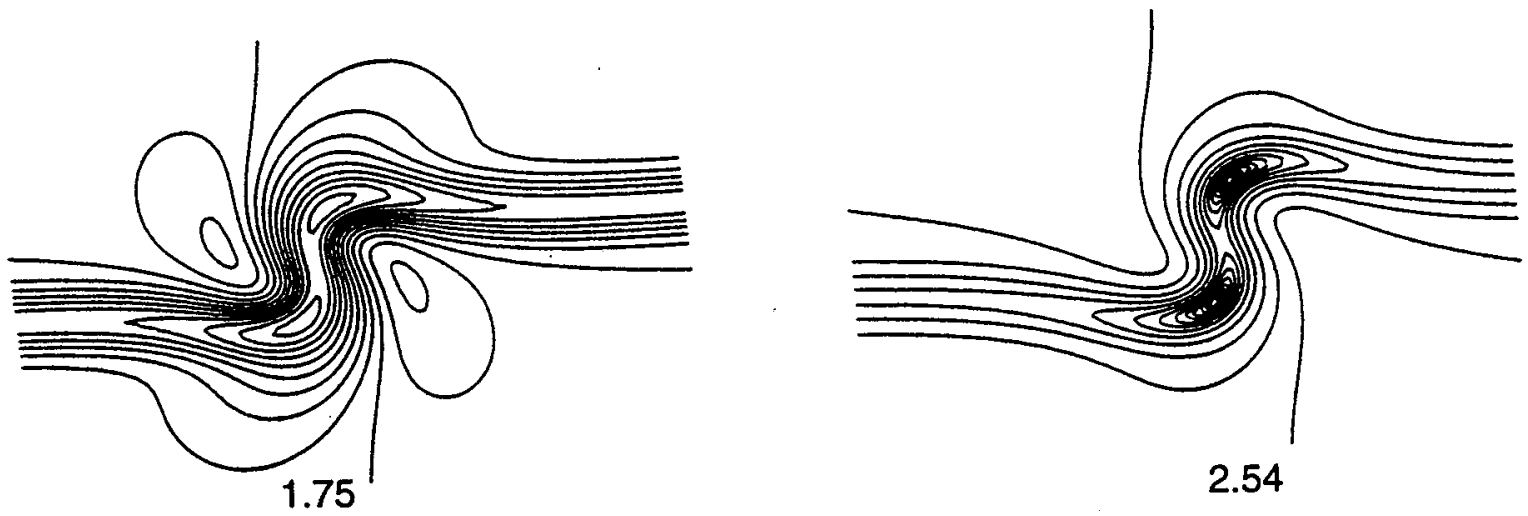

12. Contours of the temperature perturbation $\left(T_{1}\right)$ evolution from early time to ignition. $\operatorname{Re}=10, M_{0}=0.316, k_{2}=20$. 


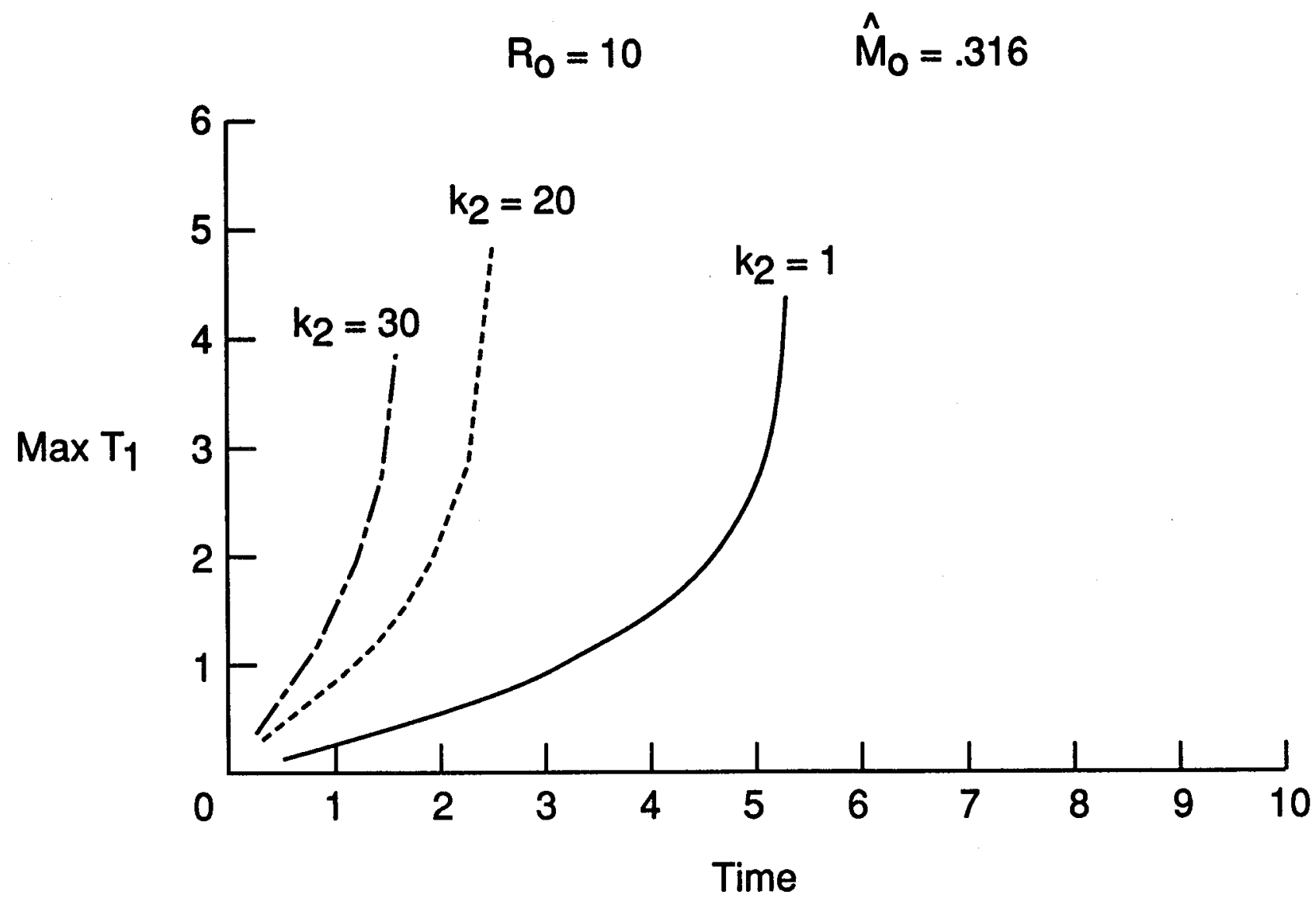

13. Plot of the maximum temperature perturbation $\left(T_{1}\right)$ versus time for various shear strengths. $\operatorname{Re}=10, M_{0}=0.316$. 


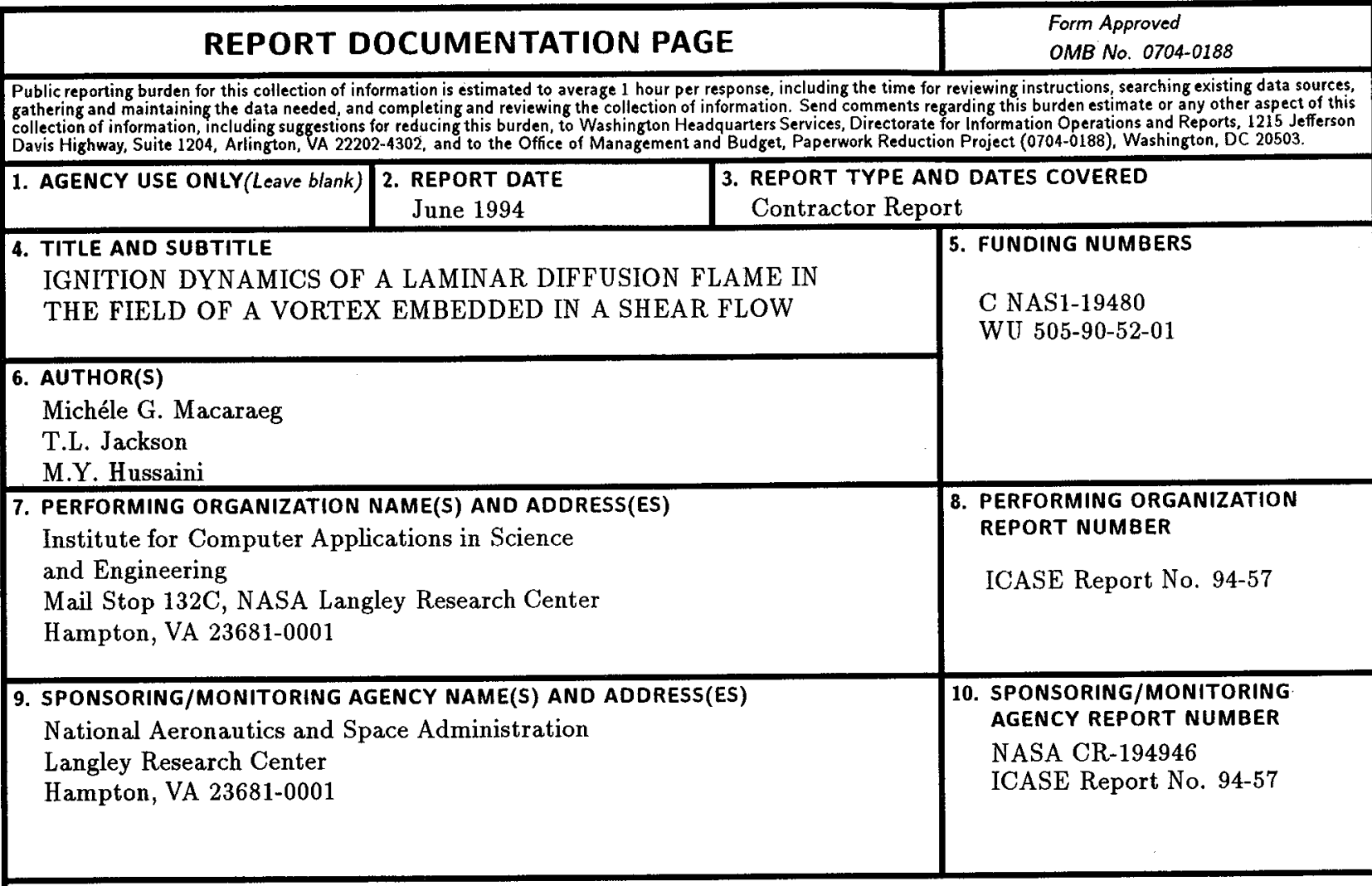

\section{SUPPLEMENTARY NOTES}

Langley Technical Monitor: Michael F. Card

Final Report

Submitted to Combustion Science and Technology

12a. DISTRIBUTION/AVAILABILITY STATEMENT

12b. DISTRIBUTION CODE

Unclassified-Unlimited

Subject Category 34, 07

13. ABSTRACT (Maximum 200 words)

The role of streamwise-spanwise vorticity interactions that occur in turbulent shear flows on flame/vortexinteractions is examined by means of asymptotic analysis and numerical simulation in the limit of small Mach number. An idealized model is employed to describe the interaction process. The model consists of a one-step, irreversible Arrhenius reaction between intially unmixed species occupying adjacent half-planes which are then allowed to mix and react in the presence of a streamwise vortex embedded in a shear flow. It is found that the interaction of the streamwise vortex with shear gives rise to small-scale velocity oscillations which increase in magnitude with shear strength. These oscillations give rise to regions of strong temperature gradients via viscous heating, which can lead to multiple ignition points and substantially decrease ignition times. The evolution in time of the temperature and mass-fraction fields is followed, and emphasis is places on the ignition time and structure as a function of vortex and shear strength.

14. SUBJECT TERMS

ignition, combustion, vorticity, asymptotics, numerics
15. NUMBER OF PAGES 34

16. PRICE CODE $\mathrm{A} 03$

20. LIMITATION OF ABSTRACT OF ABSTRACT OF THIS PAGE Unclassified

认ै U.S. GOVERNMENT PRINTING OFFICE: 1994 - 528-064/23020
Standard Form 298(Rev. 2-89) Prescribed by ANSI Std. Z39-18 298-102 